\title{
Andrés Febrés, linguista esule in Sardegna (1783 ca - 1790)
}

\author{
Andrés Febrés, an exile linguist in Sardinia (1783 ca - 1790)
}

EMANUELE PES

emanuele_pes@yahoo.it

Independent Researcher

GERTRUDIS PAYÀS

gpayas@uct.cl

Universidad Católica de Temuco

\begin{abstract}
Riassunto: L'arrivo in Sardegna dell'esule gesuita Andrés Febrés (Manresa, 1732 - Cagliari, 1790), in fuga da agenti dello Stato della Chiesa e dell'ambasciata spagnola a Roma, segna la ripresa di interessi linguistici già espressi con l'Arte de la lengua general del reyno de Chile (Lima, 1765). Privo di mezzi materiali, operando da clandestino con il falso nome di Bonifacio d'Olmi, e in parallelo a un'attività di stampa illegale, concepisce un progetto di pianificazione della lingua sarda, con una grammatica delle varianti del sardo e almeno un'opera in versi su cui sperimentare le sue innovazioni ortografiche, lessicali e morfologiche. Vorremmo ripercorrere il profilo di quell'esperimento, in buona parte interrotto dal diniego da parte delle autorità di governo dell'Isola del permesso di stampa della grammatica, di cui si sono salvate, allo stato, poche pagine manoscritte.
\end{abstract}

Parole chiave: Esilio dei gesuiti, Linguistica missionaria, Mapudungun, Lingua sarda, Metafonia, Futuro analitico e sintetico

Abstract: After his arrival in Sardinia in the first half of 80's decade, the expatriated Jesuit Andrés Febrés (Manresa, 1732 - Cagliari, 1790) seems to rediscover the linguistic interests he had already expressed with the Arte de la lengua general del reyno de Chile (Lima, 1765). In the island, hiding from Papal and Spanish agents and operating under the name of Bonifacio d'Olmi, engaged in a clandestine printing activity against the suppression of the Company, he conceived a language planning project of the Sardinian language, that included a grammar of Sardinian language varieties and at least one work in verses on which to experiment his orthographic, lexical and morphological innovations. The project largely halted as the ruling authorities in the island refused the printing permission for the grammar. Only a few handwritten pages of the grammar at the moment are known. Our purpose is to describe the outlines of Febrés' attempt.

Keywords: Jesuit expulsion, Missionary linguistics, Mapudungun, Sardinian language, Metaphony, Analytical and synthetical future

*De parte de Gertrudis Payàs, este trabajo se enmarca en el proyecto Fondecyt Regular 1170419, de la Agencia Nacional de Investigación y Desarrollo del Gobierno de Chile. 


\section{Introduzione}

Nella tarda primavera del 1787, dai torchi della Stamperia Reale di Cagliari usciva alla pubblica luce un poema in tre canti dedicato a Sant'Efisio, contenente la prima descrizione delle cerimonie e della processione che si svolgono a Cagliari il primo maggio di ogni anno, nella forma in cui più o meno si presentano ancora oggi a noi. Il testo, in sardo, per quanto provvisto delle licenze ecclesiastica e civile, appariva anonimo, ma era dell'ex gesuita catalano Andrés Febrés, missionario in Cile, e autore di una grammatica della lingua mapuche pubblicata a Lima nel 1765. Dopo l'espulsione dei gesuiti dai territori spagnoli (1767) e la soppressione canonica dell'ordine (1773) aveva maturato una contrarietà via via più risoluta a quella determinazione e, costretto alla clandestinità, in fuga da una ricerca accanita promossa dal diplomatico spagnolo a Roma, Nicolàs de Azara, tramite agenti dell'ambasciata spagnola a Roma e dello Stato della Chiesa, risiedeva da qualche anno in Sardegna con il nome di Bonifacio D’Olmi (Payàs/Pes 2020: 131-153; Mattone/Sanna 2007: 7880; Hanisch 1972: 252; Siotto Pintor 1844: III, 518; Hervás/Astorgano 2006 [1795]: 207-208).

Andrés Febrés nacque a Manresa, in Catalogna, nel 1732 ${ }^{1}$, figlio di Bonifacio Febrés e Paula Oms. Iniziato il noviziato nella Compagnia di Gesù nel 1752 a Tarragona, fu destinato alla provincia del Cile e giunse a Santiago nel 1756. Qui compì gli studi di teologia, e fu ordinato sacerdore nel 1762. Iniziato il lavoro missionario tra le popolazioni mapuche, fu incaricato dal provinciale della Compagnia di stendere la grammatica della loro lingua (Rondón 2014), la seconda dopo quella di Luis de Valdivia, la cui prima edizione era del 1606. Si trasferì a Lima nel 1764 per seguire da vicino le fasi della stampa della grammatica, che vide la luce nel 1765. A seguito dell'emanazione della Pragmática Sanción di Carlo III, nel 1767 fu arrestato e espulso, con tutti i suoi confratelli, dal Cile e dai regni spagnoli. Nel 1768 raggiunse Imola, negli Stati Pontifici, dove fu ricostituita la provincia cilena della Compagnia, sotto il nome di San Cassiano. Nel 1769 emise gli ultimi voti. Poco dopo la soppressionedell'ordine, determinata dal breve Dominus ac Redemptor di papa Clemente XIV, sciolto da vincoli formali, sembra iniziare un periodo di residenza a Roma. Nel 1778 intervenne in difesa di Francisco Javier Llampillas, nella polemica seguita alla pubblicazione del Saggio storico-apologetico della letteratura spagnuola contro le pregiudicate opinioni di alcuni moderni scrittori italiani, e nel 1780/81 era impegnato nella costruzione di un orologio solare nel palazzo del Duca Mattei (Manca di Nissa, 2021; Sabba, 2019: 187-188)², quando, coinvolto nella diffusione e forse nella stampa illegale della Memoria Cattolica, opera dell'ex gesuita italiano Carlo Borgo, che sosteneva la nullità canonica dello scioglimento dell'ordine, nel corso di una perquisizione in casa di una dama romana $a^{3}$ fu scoperto un baule contenente trenta copie di questo libro, tre copie di un

1 Ma anche 1731 e 1734, le fonti della Compagnia non offrono una data univoca.

2 Febres parla della costruzione della meridiana nella Seconda Memoria Cattolica, t. II, p. 168.

3 Il 28 giugno 1781, cfr. Febrés (1783: II, 160).

SCRIPTA, Revista internacional de literatura i cultura medieval i moderna, núm. 17 / juny 2021 / pp. 139-175 ISSN: 2340-4841· doi:10.7203/SCRIPTA.17.20909 
testo di difesa dei gesuiti della Russia Bianca ${ }^{4}$, e tra le altre cose, una cassetta di caratteri tipografici, con una pagina in quarto già composta con gli stessi caratteri e pronta per la stampa di una prosa satirica indirizzata a Nicolás de Azara. A questo punto, Febrés fugge da Roma. La proprietà del baule fu ricondotta a lui nel giro di poco tempo, e l'inizio delle ricerche nei suoi confronti segna il suo ingresso nella clandestinità. Da questo momento in poi il suo nome sarà quello di Bonifacio d'Olmi (tratto dal nome del padre e il cognome della madre), mentre la sua vera identità comincerà a essere riconosciuta solo dopo la morte, nel 1790.

\section{Clandestinità e arrivo in Sardegna}

Non sappiamo di preciso in quale anno giunse in Sardegna. Nel 1788, Nicolàs de Azara, in una lettera indirizzata al conte di Floridablanca, si diceva convinto che Febrés, dopo la sua fuga da Roma, si fosse rifugiato in un primo momento in Russia, dove Caterina II non aveva applicato il breve di soppressione dell'ordine e, successivamente, rientrato in Italia, girovagasse latitante tra Genova e Torino. ${ }^{5}$

La possibilità che fosse nascosto a Genova, o in qualche altra località della riviera, e lì godesse di possibili coperture, non è peregrina. In Liguria risiedeva la comunità di ex gesuiti che Azara finì col considerare come la più pericolosa tra quelle degli esiliati spagnoli ${ }^{6}$. Comunità che trovava forse protezione nel console generale spagnolo residente, Juan Joaquín Cornejo (Guasti 2006: 79-80). A Genova risiedeva inoltre Francisco Xavier Llampillas, che nel 1779 aveva espresso riconoscenza a Febrés per l'intervento a suo sostegno nel dibattito sulle pagine delle gazzette fiorentine, seguito alla pubblicazione del Saggio storico-apologetico della letteratura spagnola (Llampillas 1779: I, p. II, 7). Nativo di Matarò, Llampillas era stato a suo tempo novizio a Manresa, e si era trasferito in Liguria dopo lo scioglimento della Compagnia (Batllori 2001: 2400).

Per quanto riguarda Torino, invece, abbiamo un'attestazione più precisa della presenza di Febrés nella città. È infatti proprio da Torino che, il 17 dicembre 1783, una lettera a firma Bonifacio d'Olmi viene indirizzata all'editore Remondini, a Venezia, con la richiesta di acquistare una lastra

\footnotetext{
4 Dalla descrizione che ne fa Febrés, sembrerebbe trattarsi di una bozza della Lettera all'autore delle memorie sulla storia del primo secolo dei servi di Maria e degli spedalieri di s. Giovanni di Dio del gesuita sardo Francesco (o, secondo altri, Antonio) Serra, una difesa dei gesuiti della Russia bianca dall'accusa di rivolta loro da un avvocato, Giovanni Battista Zanobetti, che accusava di ribellione i gesuiti della provincia russa. La prima edizione a stampa, anonima, sembra essere del 1783. Luengo conosce l'opera: "Carta al Sr. Abogado N.N., Author de las memorias sobre la historia del primer siglo de los siervos de María, y de los de San Juan de Dios. Es una hermosissima defensa de los jesuitas rusos escrita por Antorio (sic) Serra Jesuita sardo contra un miserable folleto de Zanobetti” (Fernández Arrillaga 2002: 231; Siotto Pintor 1844: III, 416).
}

5 Archivo de la Embajada de España en Roma, Ministerio de Asuntos Exteriores, legajo 359, exp. 42, Azara a Floridablanca, 28 maggio 1788, secondo la vecchia segnatura citata in Hanisch (1969: 36): "y entonces se supo que se había ido a Rusia, pero no hallando allí lo que se imaginaba, volvió a Italia y andaba estos años foragido por Turin y Genova”.

6 “Son los peores de todos sin comparación” (Azara 2010 [1784-1804]: 695). 
di rame su cui era incisa una carta della Sardegna, opera di Paolo Santini, pubblicata da poco ma contenente una serie di errori che Febrés si proponeva di correggere ${ }^{7}$.

Il 1783 e 1784 è anche la data di pubblicazione riportata sul frontespizio dei tre tomi dell'opera clandestina di Febrés, la Seconda Memoria Cattolica. (Febrés 1783-84). Padre Luengo ebbe i libri tra le sue mani nel settembre del 1785 dopo averli cercati per lungo tempo, prestatigli in gran segreto.

Oltre alla notizia sulla matrice della carta geografica di Remondini, che fa suppore già una conoscenza diretta dell'isola, in questi tomi stampati alla macchia sono almeno tre gli elementi che riconducono alla Sardegna: il falso luogo di stampa, che fa riferimento al santuario di Bonaria di Cagliari, e alla stamperia che lì funzionò nella prima metà del '700, la Imprenta del Real Convento de Buen Ayre, che ebbe al suo attivo la stampa di piccole opere come l'orazione funebre di Joseph Manca, Difuntos que hablan, muertos que predican, del 1721. Secondo Martini (1847: 25) fu in attività fino al 1722. Tuttavia ancora nel 1750 stampò, in italiano, un dramma di Pietro Metastasio, l'Artaserse, per una rappresentazione operistica da tenersi a Cagliari nel dicembre dello stesso anno, e non si può escludere che l'attività si sia prolungata oltre. Il secondo richiamo alla Sardegna è la menzione dell'arcivescovo di Cagliari, Vittorio Filippo Melano di Portula (Febrés 1783-84: II, 132), che ritroveremo tra quanti dovranno poi decidere della pubblicazione o meno della grammatica sarda di Febrés ${ }^{8}$; infine, terzo riscontro riferibile alla Sardegna, troviamo la citazione di un proverbio in sardo che Febrés utilizza per sottolineare l'impossibilità di un evento particolare: riguardo ai sospetti “del proditorio progetto d'Indipendenza dell'America" egli ritiene tanto poco probabile la possibilità che vengano chiariti, che ne scrive, con ritmo piuttosto duro:

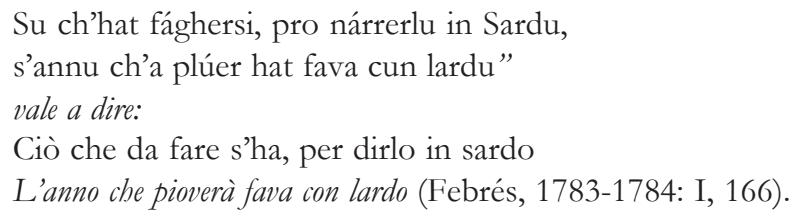

ovvero mai. Il proverbio deriva probabilmente dal Tesoru de sa Sardigna di Antonio Purqueddu, III, 65, 7-8 (Purqueddu 1779: 228): "Ma custu hat a suredi, a naiddu in sardu / Cudd'annu chi hat a proi faa cun lardu". Va anche ricordato che nel 1778 la Stamperia Reale di Cagliari stampò una silloge di proverbi sardi che accompagnava la pubblicazione il Calendario dell'anno. Ma quale che sia l'origine e il motivo di questo inserto nell'opera clandestina, esso è interessante sia perché si tratta della prima attestazione in sardo di Febrés, sia perché l'ambito paremiologico potrebbe denotare un approccio alla lingua finalizzato all'espressione sintetica, linguistica e culturale, veicolata dai proverbi e non solo al versante meramente grammaticale.

7 Biblioteca e Archivio comunale di Bassano, Epistolario Remondini, XVI, 35, 4601. Cfr. Infelise / Marini (1990: 33 ). Riproduzione della lettera in Payàs / Pes (2020: 145).

8 Su Melano di Portula, si rimanda a Palumbo (2009).

SCRIPTA, Revista internacional de literatura i cultura medieval i moderna, núm. 17 / juny 2021 / pp. 139-175 ISSN: 2340-4841· doi:10.7203/SCRIPTA.17.20909 
Altro aspetto che può aver favorito il suo arrivo nell'isola è la conoscenza di due ex-gesuiti sardi, l'algherese Michele Cubeddu, e il pattadese Giovanni Maria Cubeddu, missionari in Cile negli stessi anni in cui vi aveva operato Febrés. Michele Cubeddu, secondo l'elenco dei gesuiti della Provincia del Cile deceduti durante l'esilio, stilato dal padre Pedro Pasos (Santiago del Cile, 1743 - Imola, 1839), utilizzato da Walter Hanisch, (1972: 273) ed edito da Ferri (1997: 231), sarebbe morto a Torino nel 1786. Ma Pasos riporta anche la morte di Febrés come avvenuta, del tutto inverosimilmente, a Torino nel febbraio del 1790. È possibile quindi che Pasos abbia confuso le capitali del regno di Sardegna, cioè Torino con Cagliari. Michele Cubeddu in realtà sembra essere rientrato in Sardegna da Rimini, subito dopo la soppressione dell'ordine, trovandovi l'anziana madre come unica parente sopravissuta ${ }^{9}$. Mentre il secondo, Giovanni Maria Cubeddu, era giunto a Civitavecchia, il principale porto degli Stati pontifici, nel dicembre 1770, e attendeva, con altri gesuiti sardi provenienti dalle missioni americane, il permesso da parte del Re di rientrare nell'isola (Monti 1915: 250); nel 1778, lo troviamo nel Convitto exgesuitico di San Michele a Cagliari, da dove invia una supplica al Papa per ottenere la facoltà di confessare e predicare in quella residenza, sempre subordinato all'Arcivescovo ${ }^{10}$.

Sardo infine è l'ex gesuita Serra, che -secondo padre Luengo- fugge da Roma nel 1781 nelle stesse circostanze occorse a Febrés (Fernández Arrillaga 2003: 181), e al quale si attribuisce la stesura dell'opuscolo in difesa dei gesuiti della Russa bianca (supra: n. 4).

\section{Il quadro istituzionale e linguistico.}

La Sardegna, e i domini dei Savoia (Batllori 1966: 79; Guasti 2001: 45), non conobbero forme di residenza stabile di comunità di gesuiti iberici espulsi. Ma, come si è visto, questo non impediva che esistessero rapporti diretti e indiretti. La provincia gesuitica sarda, nonostante il passaggio dell'isola ai domini dei Savoia, era rimasta nell'Assistenza gesuitica spagnola fino al 1766, cioè fino all'anno precedente la soppressione delle province spagnole dell'ordine, determinata dalla Pragmatica Sanción di Carlo III. Del resto le regioni francofone dei domini, a partire dalla Savoia, appartenevanoalla provincia gesuitica di Lione (Signorelli 2006: 113). L'ampio processo avviato in Sardegna dal ministro Bogino, a partire dal 1760, con la riforma delle scuole e delle Università e l'introduzione dell'italiano come lingua obbligatoria d'insegnamento, aveva portato a una tensione crescente con la componente dei gesuiti sardi maggiormente legati all'appartenenza culturale iberica. Va precisato che, a differenza delle esperienze portoghese, francese e spagnola, il governo sabaudo non cercava una contrapposizione frontale con la Compagnia, alla quale (riducendone drasticamente il potere nelle scuole e nelle Università) continuava ad attribuire un ruolo "strutturale" nel campo dell'insegnamento. Sarebbe pertanto una forzatura ascrivere i Savoia a un "campo" antigesuitico a livello europeo (come rileva Storrs: 143-145).

9 ASC, Segreteria di Stato e di Guerra, I, 298, c107v, dispaccio del 7 gennaio 1774.

10 ASDC, Ordini religioni, Gesuiti, 6, f210r, 1778.

SCRIPTA, Revista internacional de literatura i cultura medieval i moderna, núm. 17 / juny 2021 / pp. 139-175 ISSN 2340-4841 · doi:10.7203/SCRIPTA.17.20909 
In Sardegna, la questione fu invece affrontata portando il confronto dentro la stessa Provincia gesuitica e inviando nell'isola gesuiti provenienti dall'Italia settentrionale, destinati in parte significativa all'insegnamento universitario. La tensione comunque persisteva, e nei suoi aspetti simbolici era sfociata nel divieto per i gesuiti sardi dell'utilizzo della berretta spagnola e più concretamente, nel 1766, nell'espulsione dal regno di due gesuiti filospagnoli, uno dei quali (il padre Santos), appena a Cagliari giunse la notizia del motín de Esquilache, secondo il viceré si rese protagonista di:

\begin{abstract}
(...) di una imprudentissima e maliziosa parlata da lui tenuta in una assemblea; ed è che sparsa qui la voce del popolare tumulto di Madrid osò il medesimo spiegarsi che là veramente v'era dello spirito, e intendevasi e sapevasi scacciare gl'Italiani: una tale proposizione avria meritato che gli dessi sul campo lo sfratto, pure non avendone io tutta quella certezza fisica per devenirvi, ho sospeso di farlo, tanto più che la breve dilazione di pochi giorni non è per sottrarlo al dovuto gastigo, e il mondo ascriverà a ben tutt'altro la di lui espulsione, onde anche per ragione politica sembrommi savia la sospensione. ${ }^{11}$
\end{abstract}

I due gesuiti furono banditi e costretti a imbarcarsi dopo poco tempo. L'espulsione del 1767 dai domini del re di Spagna stempererà enormemente le propensioni della componente filoiberica nella Provincia gesuitica sarda. E nel giro di pochi anni, la soppressione canonica dell'ordine avrebbe messo le diverse anime della Provincia definitivamente sullo stesso piano. A Cagliari, il breve papale di soppressione dell'Ordine, del 21 luglio 1773, fu ricevuto dalla cancelleria dell'Arcivescovo il 13 agosto successivo. Il 9 dicembre la Reale Udienza, dando seguito alla volontà del $\operatorname{Re}^{12}$, ne concedeva l'exequatur. Il 20 dicembre l'arcivescovo Delbecchi rese esecutiva la soppressione, con la lettura del Breve ai gesuiti radunati nel collegio di Santa Croce, nella scuola di Santa Teresa, e nella casa di probazione di San Michele ${ }^{13}$.

Anche il quadro linguistico che Febrés trova in Sardegna è quello di un'isola in rapida transizione verso l'area culturale italiana (Cadeddu 2013; Loi Corvetto 1999), che conservava sì ancora, per i patti internazionali che erano stati sottoscritti nel passaggio alla dinastia dei Savoia, la fisionomia istituzionale del regno che era appartenuto alla Corona di Aragona, ma con un'introduzione e consolidamento progressivo dell'italiano come lingua colta e di amministrazione, con una presenza della lingua spagnola, seppure ancora significativa, via via più marginale, e con la riduzione del catalano al solo utilizzo vivo nella città d'Alghero. Il ricorso al sardo, in questa dialettica tra lingue egemoni, fu promosso dai governanti sabaudi nella prima fase delle riforme sia in funzione del contenimento dello spagnolo sia per facilitare la circolazione di cognizioni tecniche, agricole ed economiche in una platea più vasta di lettori. Lo strumento principale di questa valorizzazione fu sostanzialmente l'edizione bilingue sarda e italiana di provvedimenti amministrativi e di opere didascaliche in poesia e prosa.

11 ASC, Segreteria di Stato e di Guerra, I, 27, c219r, viceré Balio della Trinità al Conte Bogino, 9 maggio 1766. 12 Sul ruolo della Reale Udienza nella concessione dell'exequatur De Giudici (2007: 136-155).

13 ASDC, Ordini religiosi, Gesuiti, 6, verbale della lettura del Breve pontificio, 1773.

SCRIPTA, Revista internacional de literatura i cultura medieval i moderna, núm. 17/ juny 2021/pp. 139-175. ISSN: 2340-4841 · doi:10.7203/SCRIPTA.17.20909 
Ma l'obbiettivo non era discuterne la condizione disubordinazione sociolinguistica. Di fronte al nuovo statuto dell'italiano, il sardo e il catalano (che conclude sotto i Savoia un percorso di marginalizzazione iniziato alla fine del Cinquecento con la progressiva castiglianizzazione politico-linguistica dell'isola) rimasero lingue proprie ma largamente destinate alla vita quotidiana, alla colloquialità, alla poesia popolare, con valenza pubblica interstiziale, se non nella catechesi e nella predicazione. E questo sia pure con l'apertura di piccoli spiragli che consentivano alle accademie poetiche un'attività letteraria nelle lingue subalterne ${ }^{14}$. Lo spagnolo non era interdetto in assoluto, ma il suo utilizzo era fortemente condizionato. E chi conosceva solo lo spagnolo vedeva preclusa la possibilità di fare carriera. È nota la vicenda di un funzionario importante come Giuseppe Cossu, che ai principii del suo impegno amministrativo subì forti critiche per la precaria conoscenza nella lingua italiana, difeso solo dal ministro Bogino che anzi vedeva in lui il segno della buona volontà di chi impara una lingua non sua. È indicativa la posizione che assume il viceré di fronte alla richiesta della Giunta exgesuitica (l'ente che si occupava di amministrare il patrimonio gesuitico dopo lo scioglimento dell'ordine) di assunzione d'un segretario per la verbalizzazione e la conservazione dei documenti:

\begin{abstract}
(...) certamente il Sig.r Lay non sarebbe al caso di compirvi colla dovuta chiarezza e precisione, poiché sebbene egli sia uomo onesto e sufficientemente instrutto nelle materie legali, tuttavia avendo fatti i suoi studi in lingua Spagnola, e ben poco coltivata l'Italiana, non saprebbe in questa spiegarsi che confusamente, e con improprietà di vocaboli, come da qualche suo scritto si è avuto luogo di rilevare (ASCA, Segreteria di Stato e di Guerra, I, 9 febbraio 1783, f262r-262v).
\end{abstract}

Le vicissitudini dell'aspirante segretario Lay, detto en passant, hanno rilievo anche nell'economia della vicenda di Febrés: quando si pose il problema di quale sostegno economico si potesse dare a Bonifacio d'Olmi / Febrés, il viceré non lo avrebbe proposto per un incarico di maestro nelle scuole inferiori se non avesse avuto una accettabile competenza nell'insegnamento in e della lingua italiana. E dell'assetto particolare del plurilinguismo in Sardegna dobbiamo tenere conto quando affrontiamo la questione dell'arrivo di Febrés nell'isola e del riemergere degli interessi linguistici che già aveva espresso con l'Arte de la lengua general del reyno de Chile (Febrés 1765).

\title{
4. L'Analisi del giudizio fatto dal giomalista fiorentino sugli scritti di Llampillas
}

In realtà questi interessi non erano sopiti neanche in precedenza. Un esempio della loro persistenza è l'opuscolo che Febrés pubblica nel 1779, Analisi del giudirio fatto dal giornalista fiorentino sul "Saggio apologetico della letteratura spagnuola" del sig. abate Saverio Lampillas, nel corso del dibattito nato con la pubblicazione dei tomi del Saggio storico-apologetico della letteratura spagnuola

14 Armangué i Herrero (2018: 7-20), che sottolinea la pratica multilingue di una timida ma sincera Arcadia, ispirata dall'ex gesuita veneto Angelo Berlendis. Sull'Arcadia catalana in Sardegna anche Armangué i Herrero (2000: 223). 
contro le pregiudicate opinioni di alcuni moderni scrittori italiani di Llampillas. Una decina di anni dopo, l'insofferente Azara avrebbe forse potuto catalogare lo scritto di Febrés tra quelli che venivano compilati per rispondere a tre finalità, tutte pericolose: “1) Por ganar la pension doble (...) 2) por introducir doctrinas, máximas y sistemas de la antigua Compañia, refrescándolas a la memoria de los españoles, y más de los indianos; 3) por hacer hablar de si en el reino (...) y hacer sentir la falta que hace en España una gente tan aplicada y tan necesaria para la instrucción" (cit. in Guasti 2006: 473). E in effetti, ultimata la stampa, Febrés inviò l'opuscolo a Floridablanca (Hanisch 1972: 277), forse anche con l'obbiettivo di una seconda pensione, della quale ad onor del vero non avrebbe potuto godere dopo la fuga del 1781. Inoltre l'opuscolo, di fronte agli attacchi del giornalista fiorentino alla letteratura spagnola a Llampillas (Giornale Fiorentino 1778: 273-275) rivendica esplicitamente la soggettività collettiva degli ex gesuiti esiliati negli Stati italiani: "E vorrete poi che stieno ritti quattromila Spagnoli?" (Febrés 1778: 10). E difende con forza Simon Nicholas Henri Linguet (ib. 12), che aveva assunto posizioni convergenti con quelle dei gesuiti (Guasti 2009: 382; Guasti 2011: 295), al quale forse Febrés guarda anche come modello di polemista.

L'opuscolo in difesa delle tesi di Llampillas parte sì dalla vexata quaestio della ricezione della cultura e della letteratura spagnola nella letteratura e cultura italiana, ma è innervato di considerazioni di natura linguistica che vengono in larga parte utilizzate per mettere in imbarazzo l'anonimo recensore fiorentino. Le evidenziamo a grandi linee: Febrés, anche piegando strumentalmente le parole ai fini della polemica, nega radicalmente al giornalista fiorentino la possibilità d'affermare che nella prosa di Llampillas "lo stile sente un poco dello Spagnuolo, e la frase non è totalmente Italiana" (Febrés 1778: 27). Gli ribatte: "Vorrete dire dell'Idioma Castigliano: sono sicuro di fissar bene questa vostra idea" (ib.), ma, e gli chiede polemicamente, come può il giornalista riconoscere un fondo castigliano nella prosa di Llampillas, quando ha implicitamente ammesso di non conoscere la lingua. E del resto, prosegue, come si riuscirebbe a provare che la frase italiana risenta di un fondo spagnolo, e non invece di uno catalano, soprattutto "essendo l'autore nativo di Catalogna?' Dunque, chiede Febrés al giornalista:

bramerei pure di vedere se saprete indovinare di qual Idioma sente il mio stile, se del Castigliano, o del Vazcuense, o del Gagliego, o Valenziano, o Catalano, ovvero d'un altro più facile dell'Italiano venticinque volte (Febrés 1778: 28).

L'idioma che sarebbe ironicamente venticinque volte più facile dell'italiano è il mapudungun. Così, dopo aver criticato il giornalista per un utilizzo improprio della sintassi del verbo passare, Febrés sottolinea tutte le forme pronominali, desinenze verbalie i solecismi utilizzati nella Gazzetta, non presenti o il cui uso era sconsigliato nella grammatica di Corticelli, ${ }^{15}$ di cui si avvale, per presentare una requisitoria finale:

15 In Sardegna Febrés utilizzerà un'edizione napoletana: Corticelli, S. (1770) Regole ed osservazioni della lingua toscana ridotte a regola, Seconda Edizione Napoletana, Napoli, nella Stamperia Abbaziana. 


\begin{abstract}
Voi smarrito, e confuso da coteste cose, e pien di smacco, vorrete per avventura diferdervi coll'appoggio, che così si usa dal volgo Toscano. Ma come? Forse gli idiotismi, e gli errori popolareschi di quella plebe ad essi avvezza, benché Toscana di nascita, e Toscanissima, hanno da dare in ciò la legge?” (Febrés 1778: 34).
\end{abstract}

Se è così, continua Febrés, anche lui sarebbe in grado di scrivere imitando gli idiotismi del popolo, e fa seguire citazioni in romanesco e romagnolo. Dunque, al di là di osservazioni in qualche caso strumentali, Febrés gioca tutta la polemica contro i critici della cultura spagnola attorno alla questione della forma standardizzata dell'italiano. Ed è estremamente significativo che questo ragionamento si tenga in un dibattito nato sulla stampa periodica, cioè sul mezzo che avrà un ruolo decisivo nello sviluppo dell'italiano standard nel corso del ‘700 (Matarrese 1993: 41-44).

\title{
5. Stampa clandestina e Seconda Memoria Cattolica
}

Per molti degli ex gesuiti contrari allo scioglimento della Compagnia, che consideravano nullo il breve d'estinzione e che cominciavano a prospettare l'ipotesi di una sua ricostituzione si presenta sempre più frequentemente la necessità di mantenere rapporti con gli ambienti della stampa e della diffusione clandestina di libri e stampati, ambienti i cui attori si muovono principalmente in funzione di un loro interesse materiale, e senza grandi pregiudiziali ideologiche.

Nel caso di Febrés, che tra l'altro ebbe sempre presenti le conseguenze che si abbatterono sugli esiliati Bruno Martí e Augustín Pujol per la stampa e la diffusione di opuscoli antiborbonici (Guasti 2006: 340-351), la propensione per la stampa clandestina è diretta, evidentemente sostenuta da una significativa perizia tecnica, le cui origini rimontano soprattutto al periodo di supervisione della stampa della grammatica del mapudungun a Lima, nel 1764. I suoi contatti peraltro orientavano verso questa scelta. Già lo scritto in difesa di Llampillas era stato stampato a spese del libraio livornese Francesco Poggiali, che proprio in quegli anni prendeva contatti anche con la Société typographique de Neuchâtel per l'importazione nel mercato romano di libri clandestini di illuministi e di libertini (Tarzia 2000: 111-118). Poggiali, al momento della scoperta delle copie della Memoria cattolica custodite da Febrés, preferì rimanere lontano da Roma, per tornare solo quando le acque si fossero calmate, almeno per lui (Febrés 1783-84: II, 170-171).

Alcune delle fonti relative al periodo di clandestinità di Febrés e a quello precedente citano il fatto che stampò "con propri torchi" (Siotto Pintor 1844: II, 113); ancora più articolato il ricordo di un gesuita Francisco Javier Mariátegui, in una sua memoria del 1812, che citiamo dall'edizione curata da Antonio Astorgano Abajo (2011: 243): “El ex jesuita Febrés, manresano, contra el sentimiento de todos, cuando lo supieron, se formó en su casa, por sí mismo secretamente, una imprenta, poco después de la abolición. Se imprimió él mismo la Memoria o promemoria que enderezó al papa Pío VI". 
La stessa richiesta della matrice in rame incisa da Santini, inoltrata a Remondini, nel dicembre del 1783 (vd. supra: 40-41), al di là della disponibilità a correggere gli errori, non sembrava avere una finalità limpidissima, e proponeva un'ambigua triangolazione con un invio della matrice a un suo uomo di fiducia a Roma che poi gliela avrebbe fatta avere (modalità tipica di operazioni illegali, e segno dei contatti che Febrés continuava a mantenere nella Città).

Ma Febrés, per quanto potesse ricercare e sapesse maneggiare materiale tipografico, era assolutamente privo di mezzi materiali ${ }^{16}$; certo, poteva far fronte alle spese della stampa clandestina grazie alle offerte che gli venivano inviate dai confratelli (lui stesso le sollecita), ma lavori come la stampa della Seconda Memoria Cattolica presuppongono il ricorso a una officina tipografica relativamente ben strutturata con compositori professionali, che difficilmente possono aver operato per una sola opera clandestina.

A testimonianza del buon lavoro "cospirativo" svolto, non sembra che Azara pensasse a Febrés come diretto stampatore della Seconda Memoria Cattolica. Credeva che vi fosse coinvolto invece un non ben identificato ex gesuita Pedro de los Campos, e che i tomi fossero stati stampati nella riviera ligure, dove Campos risiedeva (Azara 2010 [1784-1804]: 695; Guasti 2006: 472 e nn.).

Febrés naturalmente aveva tutto l'interesse a depistare le ricerche e sviare l'attenzione degli agenti dell'ambasciata e del governo borbonico. In relazione a questo obbiettivo si può spiegare il passo che segue, tratto dalla Seconda Memoria Cattolica:

Riuscitovi al fine, delusa fortunatamente la vigilanza delle nemiche sentinelle sulle stamperie, di pubblicare un'eccellente vostra difesa, quindi emanatane per violenza la proscrizione, e perseguitatine voi di nuovo, più a voi si cercherebbe di turar la bocca, di chiuder le stamperie, d'impedire la pubblicazione delle vostre apologie, più voi v'ingegnereste di moltiplicarle, e di spargerle, purché vi riuscisse, e di lamentarvi di tal nuova foggia di proscrizione e nuovo dispotismo, ben sicuri del vostro diritto senza contravenzione alle leggi: e i Gesuiti ora scimie seguaci d'un tale operar vostro ipotetico, e tanto meglio armati quanto più cresce la vostra real prepotenza e dispotismo presente, sicurissimi pur del loro diritto naturale, faran di tutto entro i limiti del giusto per pubblicare le loro Apologie e riprodurre la mal proscritta; chiuse le stamperie d'Italia, le cercheranno in paesi più liberi; chiuse ancor queste, impareranno, e forse a perfezione, l'arte tipografica, e buon saggio ne sarebbe questa prima stampa di questa medesima Opera, se ne fosse lo Stampatore un Gesuita; sì, le loro apologie stamperanno sotto terra nelle cantine, le introdurranno nelle Città Laici loro astuti e coraggiosi, travestiti da vetturali, da carbonari, o contadini; le daranno di regalo, le spargeranno, le semineranno per le strade e vie pubbliche; e per superare il maggior impossibile, che sono le spese, verrà loro alfine in mente ciò che non hanno mai finora pensato, apriran gli occhi al chiudergli al mondo i loro Socj più facoltosi; e in vece di lasciar de' legati per altre pie fondazioni, per altari, per Messe cantate, per Novenari, ed anche per le Monache, che co' lor regalucci seppero attirarsi da rimbambiti vecchj Gesuiti la corrispondenza in legati di centinaia e di migliaia di scudi, lascieranno de' legati confidenziali per quest' altro opera cento volte più pia, di stampar le difese e le Apologie della loro abbandonata madre la Compagnia; e fin gli altri men

16 Testimonianze sulla povertà di Febrés in Luengo e Hervás. 


\begin{abstract}
facoltosi, ed anche i più miserabili le Messe da lasciar ordinate per l'anime loro spartiranno con quest'opera pia, sicuri con ciò di merito e di suffragio maggiore; e forse non mancherà qualche lor divoto facoltoso che apra la mano a questo santo fine. Così vi faran sentire tutta la forza di quel principio, quod tibi non vis fieri, alteri ne feceris: non fare ad altri ciò che non vuoi si faccia a te (Febrés 1783-84: II, 220).
\end{abstract}

Il passaggio della Seconda Memoria, lasciando di fatto aperte tutte le ipotesi sulla provenienza e sul sostegno alla pubblicazione dei libri filogesuitici, assolve efficacemente al compito di disorientare le ricerche degli apparati di polizia borbonici e papalini: Febrés lascia le autorità di fronte alla verosimiglianzadi ogni ipotesi: stampare autonomamente; o fuori dagli Stati italiani, oppure in una cantina di una città italiana. Ma questo rientra perfettamente nella logica di occultamento dello stampatore alla macchia.

\title{
6. La grammatizzazione del sardo
}

Residente a Cagliari, tra la fine del 1785 e i principi del 1786, Bonifacio d'Olmi/Andrés Febrés era nelle condizioni di chiedere il permesso di stampa per una grammatica sarda: la Prima Gramatica de' tre dialetti sardi. Il pregone del 1764, che regolava l'attività editoriale e l'importazione di libri nel regnodi Sardegna, richiedeva, oltre all'imprimatur dell'autorità ecclesiastica, il rilascio di una licenza scritta da parte del Reggente la Reale Cancelleria (Editti e Pregoni: 1773: I, 117; Gabriele 2009: 93). D’Olmi/Febrés chiedeva inoltre di poter dedicare la Grammatica al re Vittorio Amedeo III.

Quali sono gli elementi che possono avere rianimato l'interesse di Febrés per la compilazione di una nuova grammatica? Il primo può sembrare banale, ma è importante ricordarlo: è l'esperienza linguistica maturata in Cile. Si potrebbe dire, rielaborando un giudizio di Manuel Alvar, sul Arte de la lengua general del Reyno de Chile (1997: 63), magari senza accettarne la componente pacificata, che l'interesse di Febrés per la standardizzazione grammaticale è una manifestazione cronologicamente estrema, di lunga durata, del lavoro linguistico impostato dal III Concilio di Lima (1583-1591). La formazione di Febrés rientra in gioco anche per il sardo e si traduce non solo in una competenza tecnica che è per molti versi già di natura professionale, ma anche in una acuta capacità di percepire $\mathrm{i}$ rapporti di subordinazione sociolinguistica che intercorrono tra lingua dominante e lingua minorizzata, e di avere strumenti linguistici, antropologici e descrittivi per proporre una lettura di questo rapporto ineguale.

Altro aspetto che ha facilitato la ripresa del lavoro di Febrés è costituito dagli studi che l'ex gesuita sardo Matteo Madao aveva dedicato al ripulimento della lingua sarda (Madao, 1782), ripulimento inteso non come semplice intento puristico, ma piuttosto come un "polire" la lingua proiettato nel futuro, come "dirozzamento, lucidatura, levigatura, perfezionamento, nobilitazione" (Lőrinci 2000: 106), verso il conseguimento di uno status di prestigio della lingua sarda. Ma c'è un'altra esperienza che Febrés può avere tenuto presente, se non come modello, almeno come stimolo 
alla ripresa del lavoro linguistico: è la sistemazione grammaticale del dialetto piemontese, considerato come a tutte gli effetti come lingua, compiuta da Maurizio Pipino, e pubblicata dalla Stamperia Reale di Torino nel 1783. Un lavoro oggi non notissimo, che tuttavia si inserì in un dibattito non banale sull'assetto plurilinguistico del Piemonte (Pilo 2015).

Va ricordato, infine, un dibattito generale a livello internazionale sulle natura delle lingue che comprendeva sia la ricerca in campo lessicale, comparativa, promossa da Caterina II di Russia tramite Pierre Simon Pallas, per l'individuazione della lingua primigenia, ricerca che fu svolta anche in Sardegna, (Mattone/Sanna 2007: 76-78); sia il dibattito nato dalle posizioni dell'olandese Cornelius de Pauw sulla maggiore o minore espressività delle lingue, sulle loro carenze lessicali o verbali, con una polemica che coinvolse in particolare gli ex gesuiti che venivano dalle Americhe, a cui Francisco Javier Clavijero, prima, e Juan Ignacio Molina, poi, rispondono direttamente (vedi infra) e a cui Febrés sembra rispondere indirettamente in un passaggio della Seconda Memoria Cattolica (Febrés 1783-1784: I, 100).

Il testo per cui Febrés chiedeva la licenza era decisamente ponderoso. Allo stato se ne conserva solo un fascicolo manoscritto ${ }^{17}$, apparentemente predisposto per stampa, contenente una pagina di occhiello di gusto simile a quelli della Stamperia reale di Cagliari, con un frontespizio ben articolato, una dedicatoria ${ }^{18}$ bilingue in sardo e italiano al re Vittorio Amedeo III, una rapida presentazione del metodo utilizzato dall'autore per compilare l'opera, e l'indice. Quest'ultimo enumera paragrafi distribuiti su 893 pagine. Forse il fascicolo è stato usato per promuovere l'associazione alle spese di stampa dell'opera. L'ìnsieme di questi paratesti superstiti rivela già a un rapido esame quanto Febrès fosse debitore nei confronti del lavoro di Madao, sia nell'accogliere l'obbiettivo del ripulimento del sardo, esplicitamente ripreso, sia nella consapevolezza degli ostacoli che si frapponevano, e che Madao aveva passato in rassegna:

I) Sembra un gittar la fatica, e un perder inutilmente il tempo l'occuparci nell'abbellire una lingua confinata alla sola Sardegna (...); II) I Sardi Dialetti sono due, quello del Capo di Cagliari, e quello del Capo di Logudoro (...); III) Ma la coltivazione del Sardo potrà impedire nel Regno la propagazione dell'Italiano; IV) Egli è disutil cosa coltivar una lingua, la quale in terra ferma è stimata barbara (...) (Madao 1782: 31-37).

È da questa condizione minorizzata definita da Madao che parte il tentativo di grammatizzazione del sardo di Febrés. Ma gli elementi strutturali della sua grammatica, nell'ambito di una strategia complessa e di compromesso, segnano un distacco dalla proposta elaborata da Madao. Li elenchiamo molto schematicamente.

17 BUC Ms.0011.02.k(10), “Prima grammatica de’ tre dialetti sardi”, c.01r-c.06r. Di seguito Febrés 1786.

18 Vd. allegato I

SCRIPTA, Revista internacional de literatura i cultura medieval i moderna, núm. 17 / juny 2021 / pp. 139-175 ISSN: 2340-4841· doi:10.7203/SCRIPTA.17.20909 
La grammatica descrive tre dialetti sardi: l'antico di Logudoro, il moderno di Logudoro, il Cagliaritano; è destinata ai giovani sardi e italiani; l'italiano non è utilizzato solo come metalingua nella descrizione della lingua oggetto, ma il consolidamento dell'utilizzo dell'italiano stesso è tra i fini della grammatica; il sardo antico rappresenta il ripulimento delle due varianti moderne.

L'impianto della grammatica è largamente descrittivo. Si tratta di un aspetto in continuità con la pratica della linguistica missionaria in generale, e dall'esperienza particolare di Andrés Febrés come compilatore della grammatica della lingua mapuche (Hernández 2019: 73; Alvar 1997). L’urgenza descrittiva è la medesima del Arte de la lengua general del Reyno de Chile:

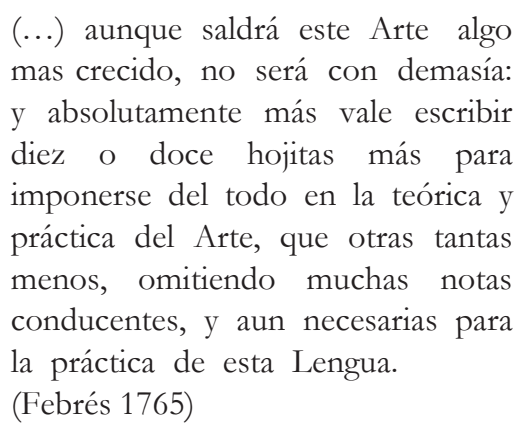

Una differenza, in realtà solo apparente, sembra emergere a causa del fatto che Febrés nel caso del sardo preferirebbe attestazioni a stampa, comunque scritte. Con tutta probabilità perché, essendo testimonianze che hanno attraversato il vaglio e la sanzione antropologica della scrittura, attribuisce ad esse un valore linguistico superiore. E del resto la pratica delle citazioni in Corticelli era questa. Presentando invece il sardo caratteristiche di lingua agrafa, compresa una scarsa produzione editoriale, non sempre gli è stato possibile trovarne. Ma il metodo che segue è lo stesso:

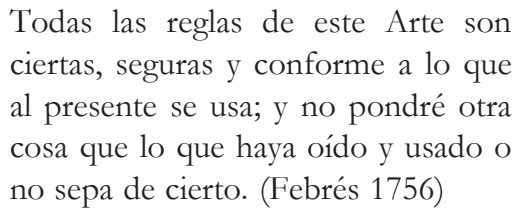
ciertas, seguras y conforme a lo que al presente se usa; y no pondré otra cosa que lo que haya oído y usado o no sepa de cierto. (Febrés 1756)

\begin{abstract}
Per altro, a cagione della grande scarsezza di libri sardi, non mi è riuscito di ritrovarne da per tutto e per ogni minuzia; nel che invece di testi bisogna contentarsi di esempli e di testimonianze intese a voce de' medesimi sardi. (Febrés 1786)
\end{abstract}

La natura descrittiva della grammatica è rafforzata dall'adozione delle Regole ed osservarioni della lingua toscana di Salvatore Corticelli come modello -non pedissequo- di riferimento. La conseguenza rilevante che questa scelta determina è una significativa delatinizzazione dell'inquadramento dei fenomeni grammaticali della lingua, con il superamento o, se vogliamo anche in termini valutativi, l'affrancamento dall'impostazione di Matteo Madao, che invece prevedeva una stretta contiguità grammaticale tra latino e sardo. 
All'interno di questo ambito descrittivo generale, Febrés ne inserisce uno storico-comparativo, legato direttamente all'obbiettivo di recuperare le testimonianze scritte della lingua: "data una regola, ordinariamente per confermarla, ove ce n'è bisogno, pongo alcuni testi d'Autori sardi, sì per il sardo antico, che per il moderno, e pel cagliaritano con quest'ordine medesimo nell'addurgli" (Febrés 1786).

Anche la scelta, in prospettiva prescrittiva, del sardo antico come standard parte da un contesto descrittivo. Mentre al di fuori da questo contesto si colloca la possibile proposta di futuro e condizionale sintetico ottenuta per analogia con altre lingue romanze, che sembra essere stata avanzata nei paragrafi 4 e 5, cap. V, del II libro dell'Indice, che in assenza del testo della Grammatica, conosciamo formalizzata esplicitamente solo nel Sant’Effisiu (Febrés 1787: 8).

Quali siano gli autori e i termini cronologici e morfologici entro cui Febrés definisce la fisionomia del sardo logudorese antico è da approfondire. I punti di riferimento espliciti sono Araolla e la Carta de Logu, e questa probabilmente nelle edizioni dei Commentaria di Olives. Ma Febrés nei suoi testi in sardo evita sistematicamente di utilizzare la -t del morfema etimologico di III plurale: risplenden, constitúin, dan (Febrés 1786); nomenan, recin, forman (Febrés 1787). Questo uso lo accosterebbe a Joan Mattheu Garipa (Legendariu, 1627) o, più vicino a lui, a Giovanni Delogu Ibba (Index libri vitae, 1736), più che proiettarlo nel Cinquecento degli autori sardi.

Dunque, in sintesi, se l'obbiettivo del progetto di pianificazione linguistica di Febrés era lo stesso di Madao (il ripulimento del sardo, la possibilità di illustrare e di conseguire il registro di "parlar polito" della lingua), sia l'analisi che gli strumenti proposti appaiono diversi. Mentre Madao punta alla contiguità del sardo con il latino ${ }^{19}$, Febrés si orienta più verso un approccio comparativo con le altre lingue romanze, con la possibilità di imprestiti non solo lessicali (la cui necessità non è affatto negata da Madao), e, come vedremo più avanti, anche morfologici (con l'inversione della forma analitica del futuro e del condizionale sardo per renderla analoga a quella della più parte delle lingue neolatine).

Febrés condivide con Madao la preminenza attribuita alla lingua italiana che, strumentale o meno, è comunque indispensabile per l'ottenimento della licenza di stampa per la Grammatica. Il testo, anche se non destinato esplicitamente agli studenti delle scuole, è indirizzato ai giovani e questa distinzione non avrebbe permesso di prescindere dal fatto che insegnamento dell'italiano e in italiano attiene alla "ragion di stato", tanto più dopo l'obbligatorietà sancita nelle scuole e nelle Università a partire dal 1760. Se per i governanti sabaudi questa "ragion di stato" non s'identificava completamente con la lingua del re concepita come segno della sovranità del monarca, come nella teorizzazione di Bodin $^{20}$ e nella pratica dell'assolutismo borbonico francese, non era per questo meno stringente. E Febrés ne era consapevole.

19 Sarebbe però un errore volere ridurre Matteo Madao alle sole sue poesie sardo-latine.

20 "On pouroit dire à plus iuste occasion, que c'est vne vraye marque de souveraineté de contraindre les sugets à changer de langue" (Bodin 1576: 182)

SCRIPTA, Revista internacional de literatura i cultura medieval i moderna, núm. 17 / juny 2021 / pp. 139-175 ISSN: 2340-4841· doi:10.7203/SCRIPTA.17.20909 
Madao, come altri autori sardi, motiva la ragione del ripulimento col dovere di natura, un'obbligazione che lega i sardi alla lingua come alla patria, ai genitori, ai fratelli. Il tema dell'obbligazione era già presente in Garipa (1627). E, se si vuole, il ricorso alla lingua locale, strumento decisivo della comprensione, era quasi sempre visto come funzionale all'assolvimento di un'obbligo: è “obligación de los rectores y curas enseñar la doctrina christiana y de los feligreses aprenderla en la lengua que entiendan" prescrivevano nel 1695 le costituzioni sinodali dell'arcivescovo di Cagliari (Turtas, 2001: 289). In Europa chi, con felice quanto prematura intuizione, la considerava un diritto da poter esercitare, come accadde nel 1685 nell'Alsazia di lingua tedesca sotto sovranità del re di Francia, alla rivendicazione: "Le roi, estimait-il, a promis par la capitulation à la ville de lui conserver tous ses privilèges, statuts et droits: l'usage de la langue est un droit" poteva sentirsi rispondere dai funzionari reali: "Il est vrai que l'usage de la lange est un droit, mais c'est un droit de la souverainité, réservé au Roi” (in Blanc 2010: 258).

Febrés, da parte sua, critica apertamente il fatto che ai rapporti di subordinazione politica (che, a scanso di equivoci, riconosce) si associno anche quelli di subordinazione linguistica. Li considera un errore politico, una mancanza rispetto ai principii di buon governo del Principe, che riduce la lingua a uno stato di "isclavitudine", alla quale oppone la "libertade" dell'espressione e del coltivamento dell'idioma: la lingua ha la sua bellesa nativa (ovviamente Febrés non motiva il suo interesse per il sardo sul dovere di natura verso la patria). Certo, l'opposizione isclavitudine / libertade della lingua riguarda anche i suoi locutori, ma non siamo ancora nell'ambito di una teorizzazione del diritto alla lingua, quanto forse del dovere della comunicazione (quella evangelica, in primo luogo, per Febrés).

Lontanissimo dalle tesi regaliste dell'arcivescovo di Città del Messico, Francisco de Lorenzana, che portarono nel 1770 all'emanazione della cédula de Aranjuez da parte di Carlo III e alla proibizione dell'uso delle lingue indigene americane, Febrés, nel non mettere in discussione le prerogative reali ma contemporaneamente nel far sentire tutto il peso dell'errore politico, sembra però vicino alle posizioni espresse da un altro ecclesiastico regalista, José Pedro Parras, nel Gobierno de regulares de América:

\footnotetext{
Nosotros tenemos en España un exemplo de lo que se puede hacer. Usamos aquí de un idioma general, y con el auxilio de las escuelas, el trato y la comunicación lo entienden todos; de modo que sin que abandonen los Gallegos, Vizcaynos, Catalanes y Valencianos el que les es natural por su primera instrucción, nos entienden y nos servimos, y comunicamos mutuamente en todo quanto se ofrece. Si esto mismo se consiguiera en los Indios, no tendriamos bastante? (...) Parece que seria util un idioma, un peso, una medida, y una moneda en cada una nación generalmente. No obstante, acá se hallan inconvenientes para establecerlo, y los mismos me persuado que se encontrarian allí (Parras 1782: II, 360-361).
}

Il paradosso di questa vicenda è che la condizione minorizzata del sardo può essere fatta risalire anche un dibattito degli anni ' 60 del Cinquecento, che ha avuto tra i suoi interpreti un gesuita che poi avrebbe partecipato al III concilio di Lima e sarebbe stato fondatore della provincia cilena 
della Compagnia di Gesù, padre Baltasar de Piñas, che fu tra quanti all'interno della Compagnia spinsero per scegliere come lingua d'insegnamento nel collegi gesuitici non il sardo, ma il castigliano, in virtù di una decisione che vedeva convergenti l'orientamento del re Filippo II e il desiderio dei genitori degli allievi che frequentavano i collegi sardi di vedere impartita una lingua di prestigio ai propri figli. Per cui la discussione fu chiusa in nome del fatto che riguardo all'insegnamento del castigliano el Rey y la tierra assí lo piden. (Turtas 1990: 233-267). Febrés con la sua grammatica tenta di riaprire quella discussione. Gli attori del processo decisionale che valuta se permettere o no la stampa della Grammatica sono: l'arcivescovo di Cagliari Vittorio Filippo Melano di Portula, il reggente la Reale Cancelleria Giuseppe Felice Giaime di Pralognan, il viceré Angelo Maria Solaro di Moretta, il ministro per gli affari interni Giuseppe Ignazio Corte di Bonvicino. L'Arcivescovo di Cagliari, che Febrés cita nella Memoria Cattolica (supra: 41), era l'unico che -con certezza- conoscesse il sardo (Turtas 1990: 133; 2001: 283). Dopo una rapida interlocuzione con il Ministero, il viceré concorderà con l'orientamento emerso da Torino: il re non era disposto ad accettare la dedica, e la licenza di stampa era negata. Per remunerare in qualche modo il suo lavoro gli viene corrisposto un compenso dalla cassa regia, e in prospettiva la possibilità d'un impiego come maestro nelle scuole inferiori ${ }^{21}$.

Questo rifiuto segna, se non il punto di svolta, il limite che i governanti sabaudi imponevano alla politica di valorizzazione del sardo in funzione del contenimento dell'utilizzo dello spagnolo. I documenti manoscritti di Febrés, compresa la Grammatica, scomparvero dopo la sua morte (Hervás 2006 [1795]). Giovanni Siotto Pintor poté consultare un sunto della Grammatica compilata a memoria da un amico di Febrés, dopo la morte di quest'ultimo, il canonico Agostino Hortal de la Bronda (Siotto Pintor 1843-1844: III, 518). Anche questo sunto è allo stato disperso.

\section{Elementi ed interpreti del dibattito sulla lingua sarda nel decennio $1780-1790$}

Gli anni che vanno dal 1780 al 1790 sono di sicura centralità per quanto riguarda il tentativo di ridiscutere lo stato di minorizzazione in cui versava lingua sarda. Forse il decennio andrebbe anticipato di un anno, per comprendervi la stampa del Tesoro della Sardegna di Antonio Purqueddu, opera didascalica in sardo e italiano dedicata alla coltivazione del baco da seta, tra le più indicative tra quelle che segnarono la promozione e l'utilizzo del sardo in quella breve stagione di disgelo linguistico su carta stampata. È praticamente certo che Febrés conoscesse il libro dell'ex gesuita Purqueddu. Non meraviglia che il suo interesse s'indirizzasse verso il lavoro di gesuiti, o verso opere che direttamente o indirettamente mettessero in buona luce la soppressa Società. Anche la sua curiosità verso gli studi di Madao era in un certo senso naturale. Febrés cita direttamente Madao nel Sant'Effisiu, e indirettamente, con l'evocazione programmatica del ripulimento, nella Grammatica. Madao non cita mai Febrés, o meglio, Bonifacio d'Olmi. Le ragioni possono essere

21 ASC, Segreteria di Stato e di Guerra, 1, c54r, dispaccio del 31 marzo; segnalato da Stefano Pira (Mattone/Sanna 2007: 79). Vd. allegato II.

SCRIPTA, Revista internacional de literatura i cultura medieval i moderna, núm. 17 / juny 2021 / pp. 139-175 ISSN: 2340-4841· doi:10.7203/SCRIPTA.17.20909 
diverse: dalla competizione intellettuale alla necessaria riservatezza con la quale proteggere un esule clandestino. Le letture di Madao erano forse un po' datate, ma non superficiali (Virdis 2014; Sanna 2006). Aveva qualche notizia della situazione linguistica nei vicereami spagnoli dell'America, e citava l'interscambio tra spagnolo e lingue amerindie e viceversa, alla pari di quelli tra inglesee tedesco, e tra francese e italiano (Madao 1782: 28). Madao non era sicuro dell'interesse del pubblico per il suo lavoro e per lesue proposte sul sardo. O, perlomeno, non era sicuro che quell'interesse reggesse economicamente il peso della stampa del suo Ripulimento e del Dirionario che aveva compilato e che era piuttosto onerosa. Arrivò così ad utilizzare uno strumento editoriale abbastanza rodato all'epoca, il "Saggio di un'opera intitolata...", che gli avrebbe consentito, anticipando i contenuti dell'opera e chiamando i lettori all'associazione alle spese, di sondare la reale fattibilità editoriale e finanziaria della stampa. Del resto, in Sardegna, lo strumento dell'associazione aveva già permesso la pubblicazione di opere particolarmente impegnative, come la Storia della Sardegna di Michele Antonio Gazano, edito dalla Stamperia Reale, sotto la direzione di Bonaventura Porro. Venne così alla luce "Il Saggio d'un'opera intitolata Il ripulimento della lingua sarda", stampato nel 1782 da Bernardo Titard, a Cagliari. Questo genere di anticipazioni erano impresse con grande cura e su buona carta per impressionare il pubblico, e anche in questo caso la consuetudine era rispettata. Andando per gradi, solo una volta stampato il Saggio, ottenne nel 1783 di poter dedicare l'opera al re Vittorio Amedeo III, a patto che la dedica non fosse "troppo ampollosa" 22 . E nondimeno, la raccolta di fondi non decollava. L'associazione minima non venne raggiunta e l'opera linguistica e lessicografica di Madao rimase allo stato manoscritto. ${ }^{23}$

Per quanto tutto lo sforzo di Madao fosse legato alla nuova consapevolezza politica che stava emergendo, le élites dirigenti che interpretarono la Sarda rivoluzione dei successivi anni '90, non sembrarono curarsi minimamente di lui e delle sue idee. Non che non conoscessero Madao, ovviamente. Segnalavano orgogliosamente il fatto che avesse dimostrato che il sardo altro non era che (quasi) schietto latino e che derivava dal greco (sic). Lo apprezzavano per le considerazioni storiche e antiquarie contenute nelle sue Dissertazioni, del 1792 (peraltro non tutte infondate). L'interesse del pubblico paradossalmente si indirizzava proprio verso la parte più debole, anche scientificamente, della sua riflessione. Ma sulla sostanza, cioè sulla novità della proposta di Madao, sul "ripulimento del sardo", cioè sulla possibilità per il sardo di acquistare il registro del "parlar polito" con un serio lavoro di limatura stilistica, calò un silenzio che forse non si può spiegare solo come disinteresse. Di fatto, in quegli anni la sua proposta di definizione dell'identità linguistica della Sardegna non venne accolta. O, meglio, non coincise con le spinte di una questione nazionale che sembrava definendosi. Da Torino, nel 1788, fu rifiutata l'idea che l'opera fosse stampata a spese pubbliche. Il ministro degli Affari interni, Conte Corte, si esprimerà con il viceré in termini abbastanza chiari: "Rispetto al Sacerdote Matteo Madau (...) perciò che riguarda la stampa della di lui opera sulla lingua sarda dedicata a S.M." bisognerà affidarsi solo ai fondi che

22 ASC, Segreteria di Stato e di Guerra, I, 303, dispaccio del 7 marzo 1783, c282r

23 BUC, fondo Baille, S. P. 6.1.39/40

SCRIPTA, Revista internacional de literatura i cultura medieval i moderna, núm. 17 / juny 2021 / pp.139-175 ISSN: 2340-4841 · doi:10.7203/SCRIPTA.17.20909 
lo stesso autore riuscirà a raccogliere, "senza impegnare la Regia Cassa nella spesa che occorrerà alla medesima". Nel 1790, il tipografo torinese Bonaventura Porro, stabilitosi definitavamente a Cagliari, avrebbe fatto l'ultimo tentativo di stampare almeno il dizionario di Matteo Madao. Chiese allo storico Giuseppe Vernazza se fosse stato possibile recuperare 300 sottoscrittori tra Piemonte, Italia e Germania che aggiunti ai 200 possibili associati sardi, avrebbero consentito la stampa dell'opera "con qualche beneficio" 24 . Ma l'iniziativa non ebbe seguito. Il progetto di Madao si era in realtà sfumato per la mancata associazione alla stampa degli anni precedenti.

Nel 1787 Madao pubblica un volumetto, che s'inserisce anch'esso nell'alveo del ripulimento della cultura e della lingua sarda, vista soprattutto in relazione a quella che Madao riteneva essere la sua origine "greca e latina": Le armonie de' Sardi. Si trattava sostanzialmente di un manuale di etnoletteratura. Le digressioni mitico-antiquarie del libro rappresentano anche qui la parte debole del lavoro, ma le attestazioni di generi, metri, pratiche poetiche e musicali, sono di rilievo significativo. Conclude l'opera un'antologia di poesie: una prima serie di poesie sardo-latine secondo il "gioco" compositivo di scrivere i versi in modo tale che si potessero leggere quasi indifferentemente nell'una o l'altra lingua. Una pratica che ebbe una qualche diffusione nella letteratura spagnola e coloniale del '600 (Lőrinczi 2000). Una seconda serie, di quarantuno "sarde poesie familiari" che invece rispecchiano un sardo più vicino all'uso poetico comune. Al di là delle forzature, l'opera fornisce un'importante documentazione delle pratiche poetiche e musicali della Sardegna del '700, generalmente riconosciuta dagli studiosi (Lavinio 1997).

Un'altra opera, del 1782, può essere ricondotta a un ambito filogesuitico. È S'Anghelu de sa Guardia, propostu in meditationes, essemplos, orationes e praticas de virtudes del sacerdote Giovanni Pinna, compendio di meditazioni e orazioni dei padri gesuiti Fabio Ambrogio Spinola e Giuseppe Antonio Patrignani, sul tema dell'Angelo custode, tradotte in sardo dall'italiano. Il testo è stampato nello stesso anno, e anche dallo stesso tipografo, Bernardo Titard, del Saggio del ripulimento di Matteo Madao, e sembra seguire argomentazioni simili. A differenza di Madao, Pinna si misura con la prosa in sardo, sollevando la questione dell'ortografia:

"comente su limbazu Sardu no est istadu pro su passadu cultivadu, né hat hapidu mai regulas fixas de sa manera de s'iscriere, hapo judicadu necessariu de narrer brevemente calchi cosa de sa naturalesa sua” (Pinna 1782: 3).

L'introduzione di Pinna rappresentarà il punto di partenza del preambolo grammaticale che Febrés inserirà nel Sant'Effisiu.

24 Archivio Storico dell'Accademia delle Scienze di Torino, Carteggio Porro, ms. 11498, lettera del 12 marzo 1790, e ms. 11501, lettera del 4 giugno 1790. Vd. Olivari (2000: 569). 
Sempre proseguendo sul filone filogesutico possiamo individuare altre due figure di sacerdoti secolari, e non provenienti dalla Compagnia di Gesù, che appaiono interessati alle questioni relative alla lingua sarda e su un altro versante sono impegnati nel sostegno alla soppressa Compagnia e alla sua storia. Entrambi promuovono la venerazione verso un gesuita cagliaritano vissuto a cavallo tra '500 e '600, Francisco Hortolà. Il primo dei due è una figura che ci è già nota. Si tratta del sacerdote che dopo la morte di Febrés compilò il sunto della Grammatica sarda, il canonico Agostino Hortal de la Bronda. È, tra le altre cose, lui che predispone per la stampa, mai realizzata, un libro Historia del siervo de Dios y venerable hermano Francisco Hortolan sardo caralitano hermano coadiutor de los RR. clerigos regulares de la Compañia de Jesús. L'altro sacerdote che, secondo Siotto Pintor, scrive in latino un trattatello in difesa di Francisco Hortolà, ed è associabile a Febrés è il cagliaritano Francesco Angelo Ferdiani, autore di una traduzione in cagliaritano della Passione di San Potito, tratta dal Martirologio romano, che si affianca al Sant'Effisiu di Febrés per il tema ma soprattutto perché il testo in sardo reca, sia pure apportato in un secondo tempo o da una seconda mano, l'accentuazione delle parole con segni che permettono si distinguere la pronuncia delle vocali aperte o chiuse, secondo le regole della metafonia enunciate da Febrés nel Sant'Effisiu, come vedremo qui di seguito. Il testo sardo della Passione di San Potito è meritoriamente edito da A. Luca De Martini (De Martini 2004: 67-72) che lo considera di anonimo. La paternità, almeno della grafia, si può stabilire e attraverso l'attribuzione che ne fa Siotto Pintor (1844: III, 525) e con il confronto con altri manoscritti di Ferdiani.

\section{Sa vida, martiriu e morte de Sant'Effisiu (1787)}

Come abbiamo anticipato, Febrés affianca al lavoro grammaticale un'opera in versi, il Sant'Effisiu, in 249 ottave, articolate in tre canti, dedicata all'arciconfraternita del Gonfalone. Il modello dell'opera, soprattutto per i primi due canti è Sa vida, su martiriu et morte dessos gloriosos martires Gavinu, Brothu et Gianuari di Araolla, stampato a Cagliari nel 1593. Il terzo canto è invece il resoconto puntuale e ricco di particolari della processione del primo maggio. Soprattutto quest'ultimo canto, che comprende anche l'esposizione dei miracoli più recenti del Santo, è più legato all'impianto narrativo e cronachistico delle relaciones de sucesos, genere che ebbe anche in Sardegna un'ampia diffusione (Paba 2012): come rileva Febrés, sono disponibili varie e evidentemente ponderose fonti sulle gesta di Sant'Efisio (i Bollandisti, Vidal, Arca), ma per il pubblico non è disponibile "relazione alcuna manuale delle gesta (...) ed affatto nessuna delle sue glorie postume" (Febrés 1787: 7). La scelta del logudorese è spiegata con il fatto di essere più comprensibile per sardi e forestieri (e qui c'è il riflesso dell'idea di lingua veicolare che già era presente nella sua Grammatica), ma anche

perché i sardi di alcune parti del capo di Logudoro, sono stati evangelizzati proprio da Sant'Efisio (Bazzano 2017: 102).

La motivazione dell'utilizzo del logudorese offre l'occasione per un'esposizione più ampia delle

SCRIPTA, Revista internacional de literatura i cultura medieval i moderna, núm. 17 / juny 2021 / pp. 139-175 ISSN: 2340-4841· doi:10.7203/SCRIPTA.17.20909 
scelte morfologiche, lessicali, ortografiche, che l'autore sente il bisogno di spiegare, perché si presentano come novità praticamente assolute. Febrés in larga parte approfitta della possibilità di questa stampa per riprendere temi e proposte già presenti nella Grammatica. Al di là di questa, però, il precedente immediato che dà a Febrés lo spunto del preambolo, è l'introduzione che il sacerdote Giovanni Pinna aveva premesso a S'Anghelu de sa Guardia, propostu in meditationes, essemplos, orationes e praticas de virtudes. Febrés parte dalle considerazioni di Pinna per presentare le sue innovazioni. Che sono ad ampio spettro. Sul piano morfologico la più rilevante è l'utilizzo del indicativo futuro semplice "come in toscano, spagnuolo e francese" ottenuto invertendo e univerbando la perifrasi del futuro sardo da hapo a timer, has a timer a Timeräpo, timeràs. Medesima soluzione per "l'altra voce del Soggiuntivo", il condizionale presente. Si tratta di una proposta che nasce probabilmente per ovviare a una critica sulla povertà del sistema verbale del sardo, suppostamente carente della possibilità di immaginare il futuro. Ne parliamo più avanti perché fu un'innovazione che in qualche caso non mancò di essere adottata nei decenni successivi alla morte di Febrés. Sul lessico, “essendo poi uno de' mezzi per arricchire di parole una Lingua il prenderle dalle altre Lingue affini" sulla scorta di un'indicazione di Madao (che solo per questo non potrebbe essere definito un purista tout court), Febrés propone l'adozione in logudorese di "Desde, parola porta dallo spagnuolo, e ammessa già nel cagliaritano, e degna di essere ammettersi nel logudorese, perché molto espressiva" (ibidem: 9; Porru 1832: s.v.); di "Istessa, usata eziandio nella Carta de Logu"; e di neologismi ottenuti per analogia con altri esiti sardidal latino, come dughe da dux, come in sardo si ha lughe e nughe da lux e nux.

È però l'ortografia il terreno sul quale Febrés dedica il maggior sforzo, con un approccio che era già presente nel Arte general de la Lengua del Reyno de Chile (Calvo-Pérez 2004: 159): tentare di conseguire una soddisfacente resa ortografica del repertorio fonetico della lingua, repertorio che sembra essere esemplato però su quello urbano/cagliaritano; la pronuncia viene descritta tramite il confronto con altre lingue, per lo più romanze: toscano, spagnolo, catalano, francese, portoghese, e infine inglese e tedesco (Febrés 1787: 10-13); è descritta la sonorizzazione fonosintattica della sibilante /s/ intervocalica, con la precisazione che questo comportamento si estende a quasi tutte le consonanti ${ }^{25}$.

Una lunga e farraginosa descrizione è dedicate alla resa grafica (e tipografica) delle vocali aperte e chiuse. Il ragionamento però culmina nella definizione delle regole della metafonia come fenomeno unitario del sardo:

Le E ed $\mathrm{O}$ si pronunzia in ogni dialetto sardo chiusa, se nel logudorese le segue immediatamente o nella sillaba susseguente un I od U, o altra E od O chiusa; altramente si proferisce aperta. (Febrés 1787: 15)

25 L'osservazione doveva essere già presente nella Grammatica: "I, $\int$. 7. Due regole per la pronuncia or rimessa [sonora], or gagliarda [sorda] di tutte generalmente le consonanti dell'alfabetto sardo" (BUC Ms. 11.2K; N. 10, c. 05r). Gagliarda e rimessa per sorda e sonora è terminologia tratta da Corticelli (1770: 322). 
L'esito ortografico complessivo raggiunto da Febrés non è così vicino a quello italiano come aveva programmaticamente dichiarato. Anche l'interpunzione adottata per il Sant'Effisiu è in parte di derivazione spagnola, stabilita dalla Real Academia, nel 1754, con l'utilizzo, non sistematico, dei doppi segni di aperturae di chiusura per l'interrogazione e l'esclamazione: “¿cal'est s’intentu tóu, / fiéru, chi tenes, hómine malvadu?" / Febrés, 1787; 95, str. 65, v. 4-5); disposizione dei caratteri che Febrés usava per la prima volta, dal momento che non erano compresi nel Arte. Alcune scelte lessicali testimoniano della volontà di conservare una relazione produttiva dello spagnolo verso il sardo. Anche nel caso del Sant'Effisiu, il risultato è dato dall'equilibrio di diverse esigenze: pianificazione ortografica del sardo, ruolo di metalingua dell'italiano, apporto dello spagnolo significativo sul versante lessicale. Minore l'incidenza del catalano, che funge prevalentemente da lingua di referenza per la descrizione della pronuncia o per l'adozione di alcune rese ortografiche.

Il modello cinquecentesco di Araolla rappresenta per Febrés un riferimento imprescindibile, sia per il contenuto dell'opera, sia per l'obbiettivo perseguito da Araolla di fondare una letteratura sarda e nobilitare la lingua che la doveva esprimere. Non si può escludere che Febrés abbia avuto di fronte anche la traduzione in castigliano del Martiriu che Joan Gavino Gillo y Marignacio aveva pubblicato a Sassari nel 1616:

\footnotetext{
Nasquer non podet da' me cosa alcuna

Digna de laude senza su favore

Tou, Re, totu a quie su Sole, et luna

S'inclinant sende d'ipsos su Factore;

Concedemi in sas ateras cust'una,

Qui conosca de Te gratia, Segnore,

Qui tesse custa tela in nov'istilu

Quale requirit deligadu filu.

(Araolla, 1593)
}

\begin{abstract}
Nacer no puede de mi cosa alguna,
Digna de loa, sin tu luz, y guia,

De Reyes Rey, por quien el Sol, y Luna,

Alumbran con sus Rayos noche, y dia.

Concedeme entre otras, sola esta una,

Que cumpla tu desseo, el alma mia,

De contar lo propuesto brevemente

Con estilo, y lenguaje competente

(Gillo y Marignacio, 1616)
\end{abstract}

\footnotetext{
Ite de laude dignu nascheriat

Da me senza s'adjudu tou dilettu

Ite mai cosa bona iscriver diat,

o ghite hit como fagher de profettu

a chie naturalesa a nudda ghiat,
} 
senza gratsia de Deus, chè a su defettu:

ottennemi inter atteras cust'una

de illuminaremi, che Sole a Luna (Febrés, 1787).

Nella scelta del tema dell'opera, in particolare della conversione dei barbaricini ad opera di Sant'Efisio, ha influito molto probabilmente una lettura della conquista come strumento provvidenziale dell'evangelizzazione. Da questo punto di vista, va ricordato che secondo la testimonianza di Agostino Hortal, raccolta da Siotto Pintor, un'altra opera in sardo di Febrés, anche questa dispersa, era "Il Messico conquistato" un lungo poema epico in ottave, basato sull'opera storica di Antonio de Solis (Siotto Pintor 1843-44: IV, 310).

\section{Dopo la morte di Febrés}

Allo stato non abbiamo un documento che attesti la morte di Andrés Febrés. Il primo riscontro semi-ufficiale che ebbero amici, conoscenti e persecutori, fu forse la notizia riportata nel numero del 5 maggio della Gazzetta Universale di Firenze:

Con bastimento arrivato [a Civitavecchia] da Caglieri si è avuta la nuova della morte in quell'Isola di Sardegna del rinomatissimo Ex-Gesuita D. Andrea Fevres [sic] Catalano, noto abbastanza per gli elogj dell'Abate Sampillus [scil. Llampillas], e non meno celebre che ricercato per l'opera Apologetica in tre Tomi in ottavo. ${ }^{26}$

La sua identità tornò allo scoperto, anche in Sardegna. Giuseppe Cossu (1799) fa il vero suo nome, con quello di Matteo Madao, tra gli studiosi della lingua sarda. Ma nel giro di non molto tempo il ricordo dell'ex gesuita, anche a causa della sua clandestinità, cominciò a affievolirsie divenne difficile associarlo al suo lavoro e alle sue opere, su cui, facendo parziale eccezione per autori come Siotto Pintor, Pietro Martini o Giovanni Spano, si ingenerò dapprima una rapida, inevitabile confusione, e infine l'oblio.

Tuttavia ci sono almeno due aspetti su cui l'operato di Febrés avrebbe fatto sentire delle ripercussioni negli anni successivi. Il primo riguarda la sua attività a sostegno della Compagnia che, come abbiamo visto, aveva avuto il suo apice con la pubblicazione della Seconda Memoria Cattolica. Nel maggio del1793, il parroco di Florinas, Gavino Sechi Bologna, invia una lettera alla prima voce dello stamento militare del Regno, il marchese di Laconi Ignazio Aymerich:

(...) Soy sardo de nacimiento; y beneficiado con la retoría de Florinas, una de las villas, que componen esta baronia de Ploague, feudo de Vuestra Exelencia; y siendo V. E. meritissimamente, como lo es, cabeza del Estamento militar de Sardeña, más que devido juzgo serme este acto. Y yo que, como Vuestra Excellencia verá, voy a relevar en este escrito el gran daño, que padece esta patria en sus derechos a lo temporal, y a lo espiritual, y en los de todos sus pueblos, y en

26 Gazzetta Universale (1790), n. 36, martedì 4 maggio, Firenze, p. 288, in ASC, Mus. Risorg., 529/5. 


\begin{abstract}
los mismos de nuestro gran monarcha, con la abolición praticada de la Compañia de Jesús, si Vuestra Excelencia lo juzgase tal, como yo lo conosco, suplicole se digne aplicar su valimiento a promover, y pedir el gran bien de su remedio, qual es el restabilimiento [sic] de los jesuitas, en todo su ser, en nuestra Sardeña (...). ${ }^{27}$
\end{abstract}

Questa richiesta va inserita nel contesto più ampio di una offensiva antigiansenista e a sostegno della soppressa Compagnia, promossa in particolare da un periodico reazionario romano, il Giornale ecclesiastico di Roma. Ma è comunque difficile immaginarla senza la diffusione nell'isola negli anni precedenti della Seconda Memoria Cattolica, che del ristabilimento faceva appunto la sua parola d'ordine, e che rappresentava la premessa ideologica delle campagne dello stesso periodico romano. Non a caso padre Luengo (Fernández Arrillaga 2003: 315) registrava nel suo diario l'impressione che il Giornale ecclesiastico avesse pubblicato, postumo, uno scritto di Febrés, la Relazione istorica e teologica del Bajanismo, del Giansenismo, e del Quesnellismo (Giornale ecclesiastico 1792). In Sardegna siamo agli esordidel triennio che avrebbe tentato di dare una risposta rivoluzionaria al gravare del feudalesimo sulla società e sull'economia e alla crisi d'autorità che il governo sabaudo stava attraversando nell'isola, periodo nel corso del quale Gavino Sechi Bologna, con altri ecclesiastici, diventerà giacobino e filofrancese (Francioni, 1999: 101-124; Virdis A. 1991: 245-329).

\title{
10. La proposta di futuro sintetico per il sardo: storia di un equivoco
}

Il secondo aspetto invece è di natura prettamente linguistica e riguarda la proposta avanzata da Febrés nel Sant'Effisiu di adozione di un futuro sintetico per il sardo, che potrebbe rappresentare una risposta alla sua supposta carenza verbale:

(...) E sopra tutto qui si vedrà spesso usato a maniera di semplice, come in toscano e spagnuolo e francese, il Futuro d'Indicativo, come Timerápo, timeràs etc. in vece di Hapo, Has a tímer, e quell'altra voce del Soggiuntivo, corrispondente alla toscana Temerei, cioè Timería, Timerías etc. in vece di Haía a tímer, o Dia, Dias tímer: il qual uso allorché si renda più steso e comune, sarà un notabile acquisto di maggior speditezza per la lingua (Febrés 1787: 8).

La proposta verrebbe già anticipata nella Grammatica dei tre dialetti sardi, di cui, ricordiamo, allo stato si conservano solo dedicatoria e indice:

5. 4. Del futuro della Lingua sarda; con una rimarchevole osservazione analoga su quello delle altre sue sorelle. n. 279

§. 5. Del secondo Imperfetto del Soggiuntivo ${ }^{28}$, ossia tempo conseguente e risolutivo del Verbo della Lingua sarda; con una simile osservazione su quello delle altre sue sorelle. n. 297 (BUC, ms.0011.02.k(10), c5v).

27 ACRS (2000 [1793-1799]: 635)

28 Equivale al condizionale presente

SCRIPTA, Revista internacional de literatura i cultura medieval i moderna, núm. 17 / juny 2021 / pp. 139-175 ISSN: 2340-4841 · doi:10.7203/SCRIPTA.17.20909 
Come Febrés constata la possibilità di invertire i costituenti del futuro sardo per analogia con l'esito della più parte delle lingue romanze, propone di generalizzarne l'univerbazione, per ottenere un futuro sintetico artificiale da apo a bennere a bennerapo. Ci sembra qui essere presente la propensione "plasmatrice" della linguistica missionaria. Ma per comprendere la possibile natura della proposta è necessario richiamare almeno a grandi linee i termini di una polemica, o di un pregiudizio, che riguardò la compiutezza delle forme verbali della lingua sarda, e specificatamente la "assenza" del futuro, con la conseguente supposta incapacità dei suoi locutori di avere cognizione della dimensione temporale dell'avvenire.

Per quanto in precedenza Matteo Madao (1782: 68) avesse già precisato i termini della questione:

Avvegnacchè la lingua Sarda , non avendo (...) futuro dell'indicativo semplice, come l'hanno i Latini, sempre si serve a formali de' verbi ausiliarj (...) uniti a quel semplice verbo, che dee significare questi tempi, già messo in modo infinito. Quindi al futuro amabit non può il Sardo avere altro tempo equivalente, se non questo: est pro amare, o bat (cioè habet sincopato) a amare; ovvero det (cioè debet sincopato) amare.

seguito da Vincenzo Porru (1811: 94)

Né questa maniera di esprimere il futuro è affatto aliena dagli Italiani, i quali in vece di dire credo che tu leggerai, dicono pure credo che tu hai da leggere (...) Anche presso i Tedeschi questo tempo manca di voce semplice, i quali per dire andrò, dicono divento andare, per mangierò, divento mangiare.

e da La Marmora nel Voyage en Sardaigne (1826):

Cette particularité [del futuro] dont on trouve les analogues dans l'allemand, l'anglais, le grec moderne, et dans d'autres langues, paraîtra moins singulière à ceux qui pensent que le futur des Italiens et des Français a passé par la même forme pour arriver à celle qu'il a maintenant. Selon cette opinion, on aurait d'abord dit: en italien, amarho, amarhai, amarba, amar avemo, ensuite amarô, amarai, amara, amaremo, et enfin amerô, amerai, amera, ameremo; et en français aimer ai, aimer as, aimer a, aimer avons, ou ons, etc., avant que de dire aimerai, aimeras, aimera, aimerons, etc.

l'opinione che fu tratta da questa particolare forma di futuro e ripetutamente ripresa fu decisamente poco lusinghiera. Charles de Saint-Severin, attaché allo stato maggiore del viceré di Sardegna Ettore Veuillet d'Yenne, nel 1821/22:

\footnotetext{
"Une particularité de cette langue est de n'avoir pas de future: ce qui at fait dire que les sardes ne s'occupaient point de l'avenir. (...) Nous avons déjà remarqué à l'article caractere et genie national, qu'il y avait de l'insouciance dans le caractere sarde". (Saint Severin 1827: 171 e n.).
}

SCRIPTA, Revista internacional de literatura i cultura medieval i moderna, núm. 17 / juny 2021 / pp. 
e ancora prima Jean-François Mimaut:

\begin{abstract}
Une particularité de cette langue c'est de manquer, dans les verbes, du temps qu'on appelle le futur. On y supplée par l'infinitif qu'on fait précéder du présent de l'auxiliaire avoir, hai. Ainsi pour exprimer ces mots il fera, ils feront, on dit hat a faghere, hant a faghere, littéralement il a, ou ils ont à faire. Cette absence du futur a fait dire que les Sardes ne s'occupaient point de l'avenir (Mimaut 1824: II, 664).
\end{abstract}

Non è improbabile, dunque, che la proposta di Febrés intervenga con l'obbiettivo di ovviare a una critica sulla carenza verbale del sardo. Non c'è dubbio invece che i gesuiti che provenivano dal Sudamerica fossero molto sensibili, e reattivi, ai giudizi sulla supposta povertà delle lingue. Juan Ignacio Molina, nella seconda edizione della sua opera sul Cile, pubblicata in italiano nel 1787, inserisce una digressione critica verso alcuni studiosi che si basano sulle testimonianze di viaggiatori, (in particolare la critica è rivolta all'olandese Cornelius de Pauw, ma non solo), e scrive:

\begin{abstract}
"Di più egli [de Pauw] mena gran romore, perchè in cotesti idiomi non sa trovare alcun vocabolo atto a significare il tempo, la durazione, lo spazio, la materia, la forma, nè verun altro e essere metafisico, o morale (...) Ma non ha fatta riflessione questo erudito Filosofo, che nel suo natio linguaggio tedesco si dice materie, e form (...) parole tolte imprestito dal latino". Siamo imparziali, e confessiamo, che tutte le nazioni, sieno Americane, Europee, o Asiatiche, sono state somigliantissime nello stato selvaggio, dal quale niuna ha avuto il privilegio di esimersi. Disapproviamo pure la trascuraggine di quelli Scrittori, che fanno le meraviglie su le lingue, e i costumi de' Selvaggj americani, come se non fossero stati mai, o non vi sieno al presente altri selvaggi nel vecchio Continente, le cui usanze, e idiomi sono egualmente riprensibili. Appena si troverà un costume fra gli Americani, che non si trovi il medesimo, o l'analogo nelle altre parti della terra. Se il Dott. Robertson avesse voluto fare questo confronto, non avrebbe avuta occasione di ponderar con eccesso la rozzezza, e stravaganza de' Selvaggi americani (Molina 1787: 306).
\end{abstract}

Molina era cileno, deportato in Italia con Febrés, con Febrés a Imola (dopodichè risiederà a Bologna). Si differenzia, e forse è perfino più infervorato di Febrés (catalano), perché è nato in America, ma il paragone tra la vita degli indiani e quella delle aree rurali e arretrate europee è già esplicito nella grammatica del mapuche di Febrés, ed è una rimodulazione di un "vecchio" tema della scuola di Salamanca nel dibattito sulla legittimità della conquista dell'America e i diritti naturali degli indios. La digressione di Molina si svolge all'interno di un "ristretto della grammatica chilese" che egli offre, dichiaratamente, sulla base del lavoro di Febrés. Ed è un sentire soprattutto condiviso tra gli ex gesuiti creoli, dal momento che Molina riprende le identiche argomentazioni (indirizzate agli identici destinatari della polemica) espresse da Francisco Javier Clavijero nella sua Storia antica del Messico (Molina 1780-81: IV, 214-226; 240262; Payàs 2010: 267). 
Anche se "non pare che il sardo abbia mai conosciuto una forma sintetica di futuro come invece accade per la gran parte delle lingue romanze" (Pisano 2011: 105; vd anche Pisano 2009) e quale che fosse l'obbiettivo della proposta di Febrés, proprio mentre il ricordo di chi ne fosse l'autore tendeva a perdersi, la possibilità che esistesse un futuro "sintetico" sardo fu presa sul serio. Nel 1840, August Fuchs (Dessau 1818-1847), giovane filologo romanzo tedesco (Malkiel 1968: 285; 1976: 15; Stussi 2006: 37), pubblicava il suo Über die sogenannten unregelmässigen Zeitwörterin den romanischen Sprachen. Come fonti per la lingua sarda aveva a disposizione tre testi. Per il campidanese, il Compendio della dottrina cristiana fatto ristampare nel 1803 dall'arcivescovo Cadello (la prima edizione era del 1777), e il Tesoro della Sardegna di Purqueddu (1779). Per il logudorese sua unica fonte era la Vida, martiriu e morte con sus glorias postumas de Sant'Effisiu di Febrés, anonimo per lui. Fuchs fu costretto a descrivere per il futuro in logudorese la coesistenza di due forme: quella cagliaritana, "analitica", e quella "panromanza", che invertiva e univa i costituenti della forma analitica in una parola. (Fuchs 1840: 198)

Giovanni Spano, nella sua Ortografia Nazionale Sarda, sempre del 1840, è invece consapevole che l'autore del Sant'Effisine della Seconda memoria Cattolica fossero la stessa persona: "un tal Bonifacio Dolmi (...)un dotto ex Gesuita (...) indurione ne sia un Sardo Logudorese proverbio che riporta [nella Seconda Memoria]" (Spano 1840: I, 104). Ma indica nelle opere di Araolla e nell'Anghelu de sa Guardia di Giovanni Pinna, i testi poetici nei quali era utilizzato il futuro sintetico (Spano 1840: 86) nella forma timerhapo, timerbàs, timerbàt ( cfr. il timerápo, timerás di Febrès 1787: 8), "Araolla e l'autore dell'Anghelu hanno cantarhapo (cfr. cantarapo Febrés 1787: 17, str. 1, v. 8), fagheràt (cfr. fagheràt Febrés 1787: str. 5 v. 8 ). Questa testimonianza è stata poi variamente ripresa fino ai giorni nostri. In realtà Spano ha sovrapposto le due figure di Araolla e dell'autore dell'Anghelu su quella di Febrés. Araolla né nel Martiriu né nelle Rimas Spirituales presenta forme sintetiche di futuro (Nocentini 2001: 390, n. 19), mentre sono attestate le forme di futuro con dèvere nella sequenza infinito - ausiliare; e quelle con hàere nella sequenza ausiliare - avverbio como - infinito (Nocentini ib.; Virdis M. 2006).

Infine, Vittorio Angius, che pure attribuiva al futuro, quando non di conio sintetico almeno nell'inversione infinito - ausiliare, un utilizzo marginale (Angius 1853: 513-514), utilizza nel Cunservet Deus su $\mathrm{R} e$, l'inno nazionale del regno di Sardegna, un notevole numero di futuri sintetici (Il Liceo 1843: 350-352 e interessante n. $)^{29}$ : provaramus, bideràs, facheràt, cederàpo, scoppiaràt che convivono con $\mathrm{i}$ futuri analitici hat a mudarsi, has essere, hamus andare, hat esser factu. In buona sostanza un prolungamento ottocentesco di quel tentativo operato dalle élite intellettuali di "nobilitazione del sardo come fattore decisivo della sua integrazione nel sistema extra-isolano dominante" (Maninchedda 2001: 185).

29 La denominazione propria dell'inno è Hymnu Sardu Nationale. L' edizione anteriore del testo che siamo riusciti a consultare, con il titolo "Hymnu militare", è sulla rivista di Torino Il Liceo, e precede di un anno la prima esecuzione pubblica dell'inno, del 1844, a Sassari, con musiche di Giovanni Gonella. 


\section{Conclusione}

Il percorso di Andrés Febrés tra i due continenti, nonostante le traversie dell'esilio, testimonia di una continuità significativa dei suoi interessi culturali e linguistici, tale da configurarsi come una prosecuzione della linguistica gesuitica, dell'unico linguista missionario che nell'arco della sua vita abbia affrontato la grammatizzazione sia di una lingua amerindia che di una lingua romanza. Sulla base della sua esperienza, Febrés penetra con particolare acutezza nelle dinamiche che sostengono i rapporti di subordinazione linguistica, in un'isola che fu tra le aree europee definite come Indias de por acá nel Cinquecento. Nella sua esperienza sarda la grammatizzazione della lingua locale rappresenta l'esito non superficiale di una vasta ricognizione linguistica, antropologica e politica, che nondimeno, per quanto dotata di strumenti relativamente avanzati, non sarà in grado dincidere sulle politiche linguistiche che generalmente accompagnano l'accentramento amministrativo degli Stati moderni nel Settecento. Questo non significa che alcuni aspetti di quell'esperienza linguistica non presentino ancora tratti di attualità. Del resto, quando ancora oggi ci si interroga se il mapudungun o il sardo siano lingua o dialetto si può ipotizzare che esistano dispositivi di subordinazione linguistica, se non universali, ancora molto comuni. Il lavoro di Febrés oggi è oggetto di una nuova attenzione. Dopo il primo inquadramento della sua attività nella Sardegna delle riforme settecentesche (Mattone, Sanna, Pira), e la ricerca tesa a collegare l'esperienza cilena con quella italiana e sarda, la figura di Febrés è stata protagonista di un'opera di narrativa che ha contribuito notevolmente alla diffusione della sua conoscenza presso un pubblico vasto (Migheli 2019). Questo maggiore interesse può portare a nuovi frutti e facilitare l'individuazione dei suoi scritti dispersi, come nel caso della recente identificazione della descrizione dell'orologio solare di palazzo Mattei, a Roma (Manca di Nissa 2021). Ultima nota: Febrés muore nell'attesa dei caratteri che gli avrebbero consentito la stampa della grammatica sarda e questo apre un interrogativo finale che costituisce anche la conclusione di questa nostro intervento. Se Febrés al momento della sua morte era in attesa dei cartatteri tipografici per la Grammatica ${ }^{30}$, la domanda che ci si pone è che scelta avesse fatto: se fossero stati rimossi gli aspetti ostativi che avevano impedito a suo tempo il rilascio del permesso della stampa della grammatica; oppure se Febrés volesse procedere come nel 1781, con la cassetta dei caratteri sequestrata nel famoso baule, cioè clandestinamente. È un interrogativo che consegniamo alla futura ricerca archivistica, con l'auspicio che si possa fare luce su una questione che allo stato rimane aperta.

30 "Ma mentre si aspettavano dal continente le lettere accentate (cosa essenzialissima nel sardo per la differenza della significazione) fu attaccato da lenta paralisia, per cui ricoverò nella casa religiosa di s. Michele, dove fini sua vita nel giorno $1^{\circ}$ d'aprile 1790” (Siotto Pintor 1844: III, 518).

SCRIPTA, Revista internacional de literatura i cultura medieval i moderna, núm. 17 / juny 2021 / pp. 139-175 ISSN: 2340-4841· doi:10.7203/SCRIPTA.17.20909 


\author{
Allegato documentale I \\ Bonifacio d’Olmi [Andrés Febrés], Prima Grammatica dei tre dialetti sardi \\ Dedicatoria al re Vittorio Amedeo III \\ Biblioteca Universitaria di Cagliari, Ms.0011.02.k(10), "Prima grammatica de’ tre dialetti sardi”, c.02v-c.04v)
}

MAGNANIMU RE:

Assa Reále Majestáde Vostra, pro éssersi generosaménte dignáda de si declaráre su PRIMU protectóre dessa Limba Sarda, si depíat offérrer de justítsia \& pro gratitúdine sa PRIMA Grammatica dessa matessi, chi depiat ésser su PRIMU Libru de compónnersi pronde mostráre su méritu de ésser cultiváda \& repulída, \& chi conténet sas promítsias dessos istúdios mios supra de issa fattos.

Chie considéret attentaménte sa grandesa de custu Reále favóre, hat admiráre dignaménte cun megus tanta magnanimidade dessa Majestáde Vostra in fágherlu; \& in custu det háver plus de su bastánte pro nárrer de ISSA unu compéndiu de totus sas laudes suas in una non vana, chi tenet ja presénte sensa la déper pordiosáre. Hoe sa Sardinha cun sa natsione e Limba Sarda, chi cun totu sa prerogativa sua de adornáre su fronte dessos Possedidores suos cun prima o nova Coróna Reále fuit tenta dae su naschiméntu pro iscláva dessas natsiones \& Limbas dominántes, s’arábiga e pisána e genovésa e catalána \& aragonésa e castilhana; hoedie peri sa Majestáde Vostra essit sa prima volta de cussa isclavitúdine, \& consíghit sa plena libertáde de cumpárrer assos foristéris cun totu sa bellésa sua natíva \& de abellíresi plus cada die, coménte sas áteras Limbas. Tale e tantu est su favóre dae sa majestade Vostra fáttuli.

Pro cussu eo non cherjo nen tenjo bisónju fágher paráula dessas áteras virtúdes Régias, chi in sa Majestáde Vostra grandeménte risplénden; prochí custa sola dignatsióne sua in favóre dessu limbátgiu sardu probat in ISSA cuddas duas calidádes, chi constitúin sa verdadéra dignidáde dessos Regnántes, sa excellénzia dessu ingéniu, ei sa grandésa dess'ánima; e mi dan totu su derétu e rexóne de póderla definíre cun su Poéta venusinu: Curantem quidquid dignum sapiente bonoque est. Proíte sa Majestáde Vostra cun sa saviesa Sua hat connótu su erróre de cudda comúne gelosía política de ténner sutta sa dominánte \& in cadénas sas Limbas dessas Provínzias novaménte adchistádas \& cun sa magnanimidáde sua in abandonárela si est mostráda superióre de ánimu a totus sos Predecessóres suos.

Ma de custa política sua veraménte cristiána ind'est ja vénnidu, \& semper plus ind'hat a vénner su effectu plus desiderábile, chi, coménte dae su fogu naschet su calóre, naturalménte inde depíat resultáre: proíte cun tale justa manéra sua de pensáre \& de operáre plus de Babu, chi non de Re, sa Majestáde Vostra si hat furadu de nou sos coros de totus sos Sardos, chi attentaménte la pondéran, \& si los hat obligádos e astríntos plus de su chi medas non arriban a créder e pensáre. Et s’áteru efféctu non minus naturále, chi eo non poto passáre in silénziu, est chi sa protecsióne dae sa Majestáde Vostra cuncédida assa Limba Sarda, \& ai custu Libru cun connoschimentu de causa, vidas sas approbatsiones ch’inde han fattu persónes meda intelligéntes, det ésser prossos Sardos sa plus manna recommendatsióne, chi de issa \& de issu se lis podíat dare prochí miren s’una cun respectu, ei s’átera cun calchi appretsiu ancóra interióre, \& pro animárelos a cultiváre \& avantatjare su idioma insóro pátriu impáre cun su italiánu; chi est su inténtu \& fine miu in háver fattu cust'Opera, \& offérrerla pro dépidu \& gratitúdine assa Majestáde Vostra: cuyas Reáles manos cun su plus profúndu respéctu s'atrévit a basáre chie desítgiat ésser

De V.S.R.M.

Su ínfimu dessos servidores suos

B. d'O.

\title{
MAGNANIMO RE:
}

Alla Vostra Real Maestà, per essersi generosamente degnata di dichiararsi il PRIMO protettore della Lingua sarda si dovea offerire di giustizia e per gratitudine la PRIMA Grammatica della medesima, che doveva esser il PRIMO Libro da comporsi per mostrarne il merito di esser cultivata e ripulita, e che contiene le primizie degli studi miei su di essa fatti. 
Chi consideri attentamente la grandezza di questo Real favore, ha degnamente ad ammirare meco tanta magnanimità di Vostra Maestà in farlo; ed in ciò avrà più del bastevole per dire di LEI un compendio di tutte le sue lodi in una non vana, che ha già presente senza doverla mendicare. Oggi la Sardegna con la nazione e Lingua sarda, che con tutta la sua prerogativa di ornare la fronte de' suoi Possessori con prima o nuova Corona Reale fu tenuta fin dalla sua nascita per ischiava delle nazioni e Lingue dominanti, l'arabica e pisana e genovese e catalana e aragonese e castigliana; oggidì per Vostra Maestà esce la prima volta da codesta schiavitù e conseguisce la piena libertà di comparire a' forestieri con tutta la sua bellezza natia, e di abellirsi più ogni dì, come le altre Lingue. Tale e tanto è il favore da Vostra Maestà fattole.

Perciò io non voglio né ho d'uopo far motto delle altre virtù Regie, che in Vostra Maestà grandemente risplendono; perché questa sola degnazione sua in favore del sardo linguaggio prova in LEI quelle die qualità, che costituiscono la vera dignità de' Regnanti, l'eccellenza dell'ingegno, e la grandezza dell'animo; e mi danno tutto il diritto e ragione di poterla definire col Poeta venusino: Curantem quidquid dignum sapiente bonoque est. Perciocché Vostra Maestà con la sua saviezza ha conosciuto l'errore di quella comune gelosia politica di tenere sotto la dominante ed in catene le Lingue delle Provincie novamente acquistate; e con la sua magnanimità in abbandonarla si è mostrata superiore d'animo a tutti i suoi Predecessori.

Ma da questa sua politica veramente cristiana n'è già venuto, e sempre più ne verrà l'effetto più desiderabile, che, siccome dal fuoco nasce il calore, naturalmente ne dovea risultare: perciocchè con tal giusta maniera sua di pensare $\mathrm{e}$ di operare più da Padre, che non da Re, Vostra Maestà si ha rubbato di nuovo i cuori di tutti li Sardi, che attentamente la ponderano, e se gli ha obbligati ed avvinti più di ciò che molti non arrivano a credere e pensare. E l'altro effetto non men naturale, ch’io non posso passare in silenzio, si è che la protezione da Vostra Maestà conceduta alla Lingua sarda, ed a questo Libro con conoscimento di causa, vedute le approvazioni, che ne han fatto persone molto intelligenti, sarà pe' Sardi la più grande raccomandazione, che di essa e di esso si poteva loro dare perché guardino l'una con rispetto, e l'altro con qualche apprezzo ancora interiore, e per animargli a cultivare ed avantaggiare l'idioma loro patrio con l'italiano insieme; che è l'intento e fine mio in aver fatto quest'Opera, ed offerirla per debito e gratitudine a Vostra Maestà: le cui Reali mani col più profondo rispetto ardisce bacciare chi desidera esser

Di V.S.R.M.

l'infimo de' suoi servitori

B. d'O.

\section{AVVERTIMENTO}

Prevenendo il lettore che, se vuol leggere agevolmente e senza inciampo la Dedicatoria e Prologo in sardo, s'imponga prima nella Ortografia, che io uso da per tutto, posta nel num. 2 dell'Opera in compendio; stimo di doverlo qui avvertire brevemente del metodo, che io seguo nel dare le regole della lingua sarda. Questo è dunque quello dal P. Salvatore Corticelli adoperato nella sua Grammatica toscana, che la trattò con sì universale applauso e lode, ancor del dottissimo Pontefice Benedetto XIV: di maniera che ciò, che nell'ultima grammatica della lingua toscana fu applaudito universalmente e lodato sopra tutte le precedenti, è stato da me imitato, per quanto il comporta il linguaggio sardo, nella prima grammatica del medesimo.

Quindi è che, data una regola, ordinariamente per confermarla, ove ce n’è bisogno, pongo alcuni testi d'Autori sardi, sì per il sardo antico, che per il moderno, e pel cagliaritano con quest'ordine medesimo nell'addurgli; e notando con carattere diverso per entro di essi la parola o parole, che ci confanno: il che un ottimo pensiere si fu del Corticelli e d'incredibile sollievo pe' lettori, ma molto trascurato nell'edizione di Napoli del 1770, onde io mi servo; siccome pure l'altro suo pensiere dell'indice delle materie in fine, pur di grandissimo giovamento per cercare e ritrovare qualche punto particolare, l'ho anch'io imitato ancor di vantaggio. Pecco io poi sovente nell'addurre tali testi anzi per eccesso che per difetto; persuaso che più e meglio insegnano molti esempi con meno spiegazione, che non molta spiegazione con pochi esempi: ma se in ciò pecco, non ci pecco impunemente; perché molta più ho durato fatica in cercare gli esempli, che in distendere le regole. Per altro, a cagione della grande scarsezza di libri sardi, non mi è riuscito di ritrovarne da per tutto e per ogni minuzia; nel che invece di testi bisogna contentarsi di esempli e di testimonianze intese a voce de' medesimi sardi. (...) 


\section{Allegato documentale II}

Dispaccio del viceré Angelo Maria Solaro di Moretta indirizzato al ministro per gli affari interni Giuseppe Ignazio Corte di Bonvicino Archivio di Stato di Cagliari, Segreteria di Stato, I, vol. 305, dispaccio del 31 marzo 1786, f54r

“(...) mi sono parsi assai plausibili ed appaganti i motivi dall'E.V. allegati per non permettere la stampa della grammatica sarda compilata dall'exgesuita Sacerdote Bonifacio D’Olmi, mentre potrebbe lo studio di tale opera alienare non solo i studenti ma eziandio le persone provette dall'applicarsi alla lingua italiana che conviene di promuovere, e d'altra parte essendo ben diversi secondo i luoghi del Regno i Dialetti dell'idioma sardo non riuscirebbe di veruna utilità l'opera suddetta: a ciò vi si aggiunga che non avendo egli fondi per anticipare la spesa della stampa, come ne venni accertato da questo Monsignore Arcivescovo vi è poca speranza di far fronte alla medesima col mezzo dell'associazione, non convenendo, nemmeno, come V. E. ha ottimamente riflettuto che nel Programma di associazione (di cui l'esito è affatto incerto) si prevenga il Pubblico d'essere detta opera dedicata a S. M.

Ho quindi stimato di fargli sentire per mezzo del Signor Reggente che sebbene fosse degna di commendazione la di lui fatica nell'aver compilata la grammatica suddetta non era tuttavia S. M. in disposizione di accettarne la dedica, e non conveniva neppure di permetterne la stampa, riservandomi a seconda del sentimento del prefato Monsignore Arcivescovo, e del Signor Reggente di fargli corrispondere qualche caritatevole sussidio, e di applicarlo altresì, stanti le notizie della di lui capacità, talento e buona condotta a qualche incumbenza di scuole inferiori per procurargli in mezzo alla sua miseria il mezzo di sostentarsi, e rimunerare in qualche modo il di lui travaglio (...)" 


\section{Fonti d'archivio, manoscritte e periodiche}

ASC $=$ Archivio di Stato di Cagliari

ASDC $=$ Archivio Storico Diocesano di Cagliari

BU-CA = Biblioteca Universitaria di Cagliari

Archivio Storico dell'Accademia delle Scienze di Torino

Archivio Remondini, Musei - Biblioteca- Archivio, Bassano del Grappa

\section{Fonti bibliografiche}

ACRS (2000 [1793-1799]) Acta Curiarum Regni Sardiniae, L'attività degli Stamenti nella "Sarda Rivolurione" (1793-1799), a cura di Luciano Carta, Cagliari, Consiglio Regionale della Sardegna, 4 vol.

Angius, V. (1853) Geografia, storia e statistica dell'isola di Sardegna, in Casalis G., Diz̨ionario storico statistico degli Stati del Re di Sardegna, XVIII ter, Torino, Maspero.

Araolla, Gerolamo (1582) Sa vida, su martiriu, et morte dessos gloriosos martires Gauinu, Brothu et Gianuari, in Caralis, per Franciscu Guarneriu Istampadore de Don Nicolau Cañellas.

Azara, J. N. (2010 [1784-1804]) Epistolario (1784-1804), Gimeno Puyol, M. D. (a cura di), Madrid, Castalia.

Bodin, J. (1576) Les Six Livres de la République, Paris, chez Jacques du Puy.

Bolland, Jean (1643) Acta Sanctorum, Antuerpiae, apud Ioannem Meursium.

Clavijero, F. J. (1780-81) Storia antica del Messico: cavata da' migliori storici spagnuoli, e da' manoscritti, e dalle pitture antiche degl' Indiani, Cesena, Biasini, 4 vol.

Cossu, G. (1799 [2000]) Descrizione geografica della Sardegna, Genova, dalla stamperia di Agostino Olzati.

Corticelli, S. (1770) Regole ed osservazioni della lingua toscana ridotte a regola, Seconda Edizione Napoletana, Napoli, nella Stamperia Abbaziana.

Editti e pregoni, (1775) Editti, pregoni, ed altri provvedimenti emanati pel Regno di Sardegna dappoichè passò sotto la dominazione della Real Casa di Savoia sino all'anno 1774, Cagliari, Stamperia Reale, 3 vol.

Febrés, A. (1765) Arte de la lengua general del reino de Chile, Lima, en la calle de la Encarnación.

- (1778), Analisi del gindizio fatto dal giornalista fiorentino sul "Saggio apologetico della letteratura spagnuola” del sig. abate Saverio Lampillas, Cosmopoli [Siena], Pazzini.

- (1783-1784) Seconda Memoria Cattolica, contenente il trionfo della fede e chiesa de monarchie monarchie e della compagnia di Gesu e sue apologie con lo sterminio de lor nemici, s.l., NuovaStamperia Camerale di Bonaria, 3 vol. 
- (1787) Vida, martiriu e morte cun sas glorias postumas de Sant'Effisiu, protettore de Caralis, Caralis, in sa Imprenta Reale.

Hervás y Panduro, L. (2006 [1795]) Biblioteca Jesuítica Española, Cuenca, Diputación Provincial, Ed. de A. Astorgano.

Il Liceo (1843) giornale di scienze e di letteratura d'arti, di teatri e di mode, anno primo, Cassone e Marzorati, Torino.

Garipa, I. M. (1627) Legendariu de Santas Virgines, et Martires de Iesu Cristu, per Ludovicu Grignanu, Roma.

Gazæetta Universale (1790) n. 36. Martedì 4 maggio 1790, Firenze, p. 288.

Giornale Ecclesiastico di Roma (1792), Tomo IV, Roma, nella Stamperia di Giovanni Zempel, pp. 445513 pp.

Giornale Fiorentino (1778) Giornale Fiorentino Istorico-politico e letterario per l'anno 1778, Firenze, luglio, p. 273-275.

Gillo y Marignacio, J. G. (1616) El triumpho y martyrio esclarecido de los Illustriss. SS. Martyres Gavino, Proto y Ianuario, Sácer [Sassari], en la Emprenta del Ill. y Reverendiss. Señor D. Ant. Canopolo.

Giomale Ecclesiastico di Roma (1793) Roma, nella Stamperia di Giovanni Zempel, Tomo V, pp. 3-49.

Iturriaga, J. M. de, SJ, (1740 [2019]) Californiada. Épica sagrada y propaganda jesuítica en Nueva España, a cura di Fernando Navarro, Huelva, Universidad de Huelva.

Lorenzana, F. A. (1770) Cartas pastorales y edictos del Illustr.mo Señor Don Francisco Antonio Lorenzana, y Buitrón, Arzobispo de México, México, Imprenta del Superior Gobierno.

Madao, M. (1782) Saggio d'un'opera intitolata Il ripulimento della Lingua Sarda, Cagliari, Titard.

Mimaut, J. F. (1825) Histoire de Sardaigne, Paris, Blaise-Pélicier, 2 vol.

Molina, J. I. (1787) Saggio sulla storia civile del Chili, Bologna, nella Stamperia di San Tommaso d'Aquino.

Parras, P. J. (1783) Gobierno de los regulares de la América, ajustado religiosamente a la voluntad del Rey, Madrid, Ibarra, 2 vol.

Saint-Severin, C. de (1827) Souvenirs d'un séjour en Sardaigne pendant les années 1821 et 1822, Ayné Frères, Lyon.

Siotto Pintor, G. (1843-1844) Storia Letteraria di Sardegna, Cagliari, Tipografia Timon, 4 vol.

Spano, G. (1840) Ortografia Sarda Nationale o sia Gramatica della lingua logudorese paragonata alla italiana, Cagliari, nella Reale Stamperia, 2 vol.

Porru,V. R. (1811) Saggio di gramatica sul dialetto sardo meridionale, Stamperia Reale, Cagliari. . (1832) Nou dirionariu universali sardu-italianu, Casteddu, in sa tipografia arciobispali, [ried. a cura di Marinella Lőrinczi, Ilisso, Nuoro, 2002] 
Purqueddu, A. (1779) Il Tesoro della Sardegna ne’ bachi e gelsi, poema sardo e italiano, Cagliari, Stamperia Reale.

Valdivia, Luis de (1606) Arte y gramática general de la lengua que corre en todo el reyno de Chile. Con un vocabulario y confesionario, Lima, Francisco del Canto.

Vidal, S. (1647) Annales Sardiniae, pars III, Mediolani, ex typographia Io. Petri Cardi, sub signo Fortunae.

\section{Bibliografia}

Alvar, M. (1997) "La gramática mapuche del padre Febrés (1765)", in Alvar, M., Nebrija y estudios sobre la Edad de Oro, Madrid, CSIC, 1997, pp. 63-85.

Ambrasi, D. (1979) Riformatori e ribelli a Napoli nella seconda metà del Settecento. Ricerche sul giansenismo napoletano, Napoli, Luigi Regina.

Armangué i Herrero, J. (2000) "Sis segles en català" in Revista de filología románica: Lenguas minoritarias en la romania. El sardo. Estado de la cuestión. $N^{\circ}$ 17, 2000.

- (2018) "Multilinguismo e poesia arcadica in Sardegna. Il magistero di Angelo Berlendis" in Quaderni del Dipartimento di Filologia, Letteratura, Linguistica dell'Università di Cagliari, 17 (2018), p. 7-20.

Astorgano Abajo, A. (2011) "Un jesuita expulso sangüesino rebelde: Francisco Javier Mariátegui, el ex jesuita oprimido", in Príncipe de Viana, LXXII, 252, Enero-Abril, Pamplona, Institución Príncipe de Viana.

—. (s.d.) "Febrés Oms, Andrés", in Real Academia de la Historia, Diccionario Biográfico electrónico, http://dbe.rah.es/biografias/20279/andres-febres-oms

Batllori, M. (1966) La cultura hispano-italiana de los jesuitas expulsos : Españoles, Hispanoamericanos, Filipinos, 1767-1814, Madrid, Gredos.

- (2001) "Llampillas (Llampilles, Lampillas), Francisco Javier" in O’Neill, C. E. / Domínguez, J. M. (a cura di), Diccionario histórico de la Compañía de Jesús, Madrid, Universidad Pontificia Comillas, 4, p. 2400.

Bazzano, N. (2017) "Efisio martire: un santo contro la peste barocca nella Cagliari del Seicento", in Chronica nova, Revista de historia moderna de la Universidad de Granada, Santidad y politica: modelos de santos y su vinculo con el poder en la monarquía hispana: siglos XVI y XVII, n. 43, Granada, Universidad de Granada, pp. 85-108

Blanc, A. (2010) La langue du Roi est le français. Essai sur la construction juridique d'un principe d'unicité de langue de l'Etat royal (842-1789), Paris, Harmattan.

Birocchi, I. (1990) "Il Regnum Sardiniae dalla cessione dell'isola ai Savoia alla Fusione perfetta", Guidetti, M. (a cura di), Storia dei sardi e della Sardegna. L'età contemporanea, Milano, Jaka Book, pp. 175-213. 
Burrieza Sánchez, J (s.d.) "Baltasar Piñas", in Real Academia de la Historia, Diccionario Biográfico electrónico, http://dbe.rah.es/biografias/20890/baltasar-pinas

Calvo-Pérez, J. (2004) "Fonología y ortografía de las lenguas indígenas de América del Sur a la luz de los primeros misioneros gramáticos", in Zwartjes, O. / Altman, C. (a cura di) Missionary Linguistics II / Lingüistica misionera II, Orthography and Phonology. Selected papers from the Second International Conference on Missionary Linguistics, São Paulo, 10-13 March 2004, Amsterdam/Philadelphia, pp. 137-170.

Cadeddu, M. E. (2013) "Scritture di una società plurilingue: note sugliatti parlamentari sardi di epoca moderna”, in Krefeld, T. / Oesterreicher, W. / Schwägerl-Melchior, V. (a cura di) Reperti di plurilinguismo nell'Italia spagnola (sec. xvi-xvii), Berlin / Boston, De Gruyter, pp. 19-24.

Cogliani, T. (1908) “L'espulsione dei gesuiti dalla Sardegna”, in Archivio storico sardo, 1907.

Company Company. D, (2006) Sintaxis histórica de la lengua española, Fondo de Cultura Económica (Mexico), Universidad Nacional Autónoma de México

Corda, M. (2005) Sardae Patronus Insulae. Il culto di Sant'Efisio attraverso i secoli. Cagliari, Cuec

De Giudici, G. (2007) Il governo ecclesiastico nella Sardegna sabauda, Napoli, Jovene editore.

De Martini, A. L. (2003) “Sa vida de Santu Potitu” in NAE, anno II, numero 5, Inverno 2003, pp. 67-72.

Fernández Arrillaga, I. (2002), Éxodo y exilio de los jesuitas españoles según el diario inédito del p. Luengo (1767-1814), Alacant - Alicante, Universitat d'Alacant - Universidad de Alicante.

Fernández Arrillaga, I. (2003) El legado del padre Manuel Luengo, S.J., Alicante

Francioni, F. (1999) "Réfugiés politiques et diplomates pour l'institution d'une république sarde sous la protection de la France (1796-1800)", in Les Cabiers de la Méditerranée, Bonaparte, les îles méditerranéennes et l'appel de l'Orient, Actes du Colloque d'Ajaccio 29-30 Mai 1998, numero tematico, 57, Nice, Centre de la Mediterranee moderne et contemporaine https://doi.org/10.3406/ camed.1998.1228

Ferri, A. (1997) I gesuiti a Imola e le scuole cittadine nel complesso di Sant'Agata. Documenti, Bologna University Press, Bologna.

Gabriele, N. (2009) Modelli comunicativi e ragion di stato. La politica culturale sabauda tra censura e libertà di stampa, 1720-1852, Firenze, Polistampa, Fondazione Spadolini - Nuova antologia.

Granata, G. (2019) 'La 'rivoluzione delle idee' in Sardegna alla fine del Settecento. Le acquisizioni della Biblioteca Universitaria di Cagliari”, in Granata, G. (a cura di) Biblioteche e saperi. Circolazione di libri e di idee tra età moderna e contemporanea, Roma, Edizioni di Storia e Letteratura, pp. 39-77.

Guasti, N. (2006) L' esilio italiano dei gesuiti spagnoli. Identità, controllo sociale e pratiche culturali (17671798), Roma, Edizioni di Storia e Letteratura.

- (2009) Giudizi e pregiudiz̨i:percezione dell'altro e stereotipi tra Europa e Mediterraneo : atti del seminario, Firenze, 10-14 giugno 2008, 1, pp. 339-393. 
. (2011) "Catholic civilization and the evil savage: Juan Nuix facing the Spanish 'Conquista' of the New World", in Abbattista G. (a cura di), Encountering Otherness. Diversities and Transcultural Experiences in Early Modern European Culture, pp. 285-302.

—. (2017) Juan Andrés e la cultura del Settecento, Milano /Udine, Mimesis.

Hanisch Espíndola, W (1969) "Los jesuitas y la independencia de América, y especialmente de Chile", in Boletín de la Academia Chilena de la Historia, No 82, 1969, pp. 13-82.

—. (1972) Itinerario y pensamiento de los jesuitas expulsos de Chile, 1767-1815, Santiago del Chile, Andrés Bello.

Hernández, E. (2019) "El impacto de las lenguas indígenas en los vocabularios jesuitas (Siglo XVIII)", in Lingüistica misionera: aspectos lingüisticos, discursivos, filológicos y pedagógicos. CerrónPalomino, R /, Álvaro Ezcurra Rivero, R. A. / Zwartjes, O. (a cura di), Lima, Fondo Editorial de la Pontificia Universidad Católica del Perú, pp. 69-87.

Infelise M. / Marini P. (1990) Remondini, un editore del Settecento, Milano, Electa.

Lavinio, C. (1997) intr. a Madao, M. [1787] Le Armonie dei Sardi, Nuoro, Ilisso.

Loi Corvetto, I. (1999) "La Sardegna plurilingue e la politica dei Savoia", in Sala Di Felice / Loi Corvetto, I. (a cura di) Lingua e letteratura per la Sardegna sabauda tra ancien régime e restaurazione. Roma, Carocci, pp. 45-69.

Lőrinczi, M. (2000) Storia della lingua sarda secondo i falsi di Arborea e il concetto di "arcaicità del sardo", in Actes du XXII Congrès International de Linguistique et de Pbilologie Romanes (Bruxelles,luglio 1998), Tübingen/Tubinga, Niemeyer, 2000, I vol., pp. 105 -111.

Malkiel, Yakov (1968) “August Fuchs (1818-47), the Founder of Comparative Romance Dialectology”, in Romance Philology, XXI (1967-1968), p. 285.

Malvestitti, Marisa / Payàs, Gertrudis (2016) "Circulaciones intertextuales del Arte de Febrés a ambos lados de los Andes”, en María Andrea Nicoletti, Andrés Núñez y Paula Núñez (a cura di), Araucania-Norpatagonia III: Discursos y representaciones de la materialidad, Bariloche, Universidad Nacional de Río Negro, pp. 286-309.

Manca di Nissa, M. (2021) “Andrés Febrés S.J. e l'orologio solare del Duca Mattei”, in Accademie e Biblioteche d'Italia, Roma, numero unico 2021, in corso di stampa.

Mattone, A. / Sanna, Piero (1990) I Simon, una famiglia di intellettuali tra riformismo e restaurazione, Roma, Ministero per i beni culturali e ambientali.

Maninchedda, P. (2001) "Nazionalismo, cosmopolitismo e provincialismo nella tradizione letteraria della Sardegna”, in Revista de filología románica, No 17, 2000, numero dedicato a: Lenguas minoritarias en la romania. El sardo. Estado de la cuestión, pp. 171-196

Mattone, Antonello (2004) "Assolutismo e tradizione statutaria. Il governo sabaudo e il diritto consuetudinario del Regno di Sardegna (1720-1827)", in Rivista storica italiana, Vol. 116, N. 3, 2004, págs. 926-1007. 
—. / Sanna, Piero (2007) Settecento sardo e cultura europea. Lumi, società, istituzioni nella crisi dell'Antico Regime, Milano, Franco Angeli.

Martini, P. (1847) Sulle vicende tipografiche in Sardegna, [Cagliari], Timon.

Migheli, N. (2019) La grammatica di Andrés Febrés, Cagliari, Arkadia

Monti S.J., A. (1915) La compagnia di Gesù nel territorio della Provincia torinese, 1915, vol. II, p.

Nocentini, Alberto (2001) "La genesi del futuro e del condizionale sintetico romanzo", Zeitschrift für romanische Philologie, 117, 3, pp. 367-401.

Olivari, T. (2000) "L’editoria sarda nel Settecento", in Studi Storici, Anno 41, No. 2, pp. 533-569.

Paba, T. (2012) Relaciones de sucesos sulla Sardegna (1500-1750), Cagliari, CUEC.

Palumbo, P. (2009), voce "Melano, Vittorio Filippo", in Diærionario Biografico degli Italiani, vol. 73, Roma, Treccani.

Payàs, G. (2010) El revés del tapiz. Traducción y discurso de identidad en la Nueva España (1521-1821), Madrid / Frankfurt am Main / Temuco, Vervuert / Hiberoamericana / Ediciones Universidad Católica de Temuco, Madrid https://doi.org/10.31819/9783954871759

_., (2012) "Al mapudungun por el catalán: la obra del jesuita expulso Andrés Febres (Manresa 1734-Cagliari 1790) en Chile”, in Lafarga, F. / Pegenaute, L.(a cura di) Lengua, cultura y politica en la bistoria de la traducción en Hispanoamérica, Vigo, Academia del Hispanismo, pp. 173-180.

__. / Zavala, José Manuel / Curivil, Ramón, (2015) “La palabra 'parlamento’ y su equivalente en mapudungun en los ámbitos colonial y republicano. Un estudio sobre fuentes chilenas bilingües y de traducción”, in Historia, n. ${ }^{\circ}$. 47, vol. II, Santiago, 2015, pp. 355-373.

— - / Pes, E. (2020) "Como uno que yo me sé". Nuevos aportes a la biografía y obra de Andrés Febrés, S.J. (Manresa, 1732-Cagliari, 1790), in Historia, Santiago, vol.53, n.1, pp.131-153.

Pilo, R. (2015) "Pipino, Maurizio" in Dizionario Biografico degli Italiani, vol. 84, Treccani, Roma.

Pira, S. (2019) “Costruire un'élite per due patrie. Da Collegio dei Nobili a Convitto Nazionale di Cagliari”" in Murgia G. (a cura di), Convitto Nazionale di Cagliari. Giovani, da quattro secoli 1618-2018, Cagliari, pp. 91-101, Cagliari, Convitto Nazionale.

Pisano, S. (2009) "Il futuro e il condizionale analitici in alcune varietà sarde moderne: genesi di marche grammaticali da forme verbali lessicalmente piene" in Bollettino di Studi Sardi, 2, Cagliari, CUEC.

(2011) "Ancora sul futuro e il condizionale: casi particolari nella Sardegna centromeridionale" in Bollettino di Studi Sardi, 4, Cagliari,CUEC.

Ricuperati, G. (1986) "Il riformismo sabaudo settecentesco e la Sardegna. Appunti per una discussione", in Studi Storici, Anno 27, 1, pp. 57-92 Id., I volti della pubblica felicità. Storiografia e politica nel Piemonte settecentesco, 1989 
Rondón, V. (2014) "Havestadt v/s Febrés. A propósito de una carta y unas canciones" in Revista de Historia Social y de las Mentalidades, v. 18, n. 2, 2014, p. 79-103.

Sabba, F. (2019) Angelo Maria Bandini in viaggio a Roma (1780-1781), Firenze, Firenze University Press, 2019

Sánchez-Albornoz, N. (2001) "De las lenguas amerindias al castellano. Ley o interacción en el período colonial" Colonial Latin American Review, 10:1, pp. 49-67 https://doi. org/10.1080/10609160120049335

Sanna, P. (2006) "Matteo Madao" in Dizionario biografico degli Italiani, Treccani, Roma, vol. 67.

Signorelli, B. (2006) "I gesuiti sabaudi durante la soppressione" in Bianchini, P. (a cura di) Morte e resurrezione di un ordine religioso. Le strategie culturali ed educative della Compagnia di Gesù durante la soppressione (1759-1814), Milano, Vita e pensiero, pp. 109-131.

Storrs, C. (2015) "The Suppression of the Jesuits in the Savoyard State", in Burson, J / Wright, J (a cura di), The Jesuit Suppression in Global Context: Causes, Events, and Consequences,. Cambridge, Cambridge University Press, pp. 139-160, doi:10.1017/CBO9781139344135.008

Stussi, A. (2006) "Preistoria degli studi sul volgare padovano: una breve divagazione", in La cultura volgare padovana nell'età del Petrarca, p. 27-48

Tarzia, F. (2000) Libri e rivoluzioni: figure e mentalità nella Roma di fine ancien régime, 1770-1800, Milano, Franco Angeli.

Turtas, R. (1990) "La chiesa durante il periodo sabaudo", in Guidetti, M. (a cura di.), Storia dei sardi e della Sardegna, Milano, Jaca Book, vol. IV, p. 133

—. (2001), Studiare, istruire, governare, Sassari, EDES - Editrice Democratica Sarda. La formazione dei letrados nella Sardegna spagnola.

- (2009) "Gesuiti sardi in terra di missione tra Seicento e Settecento", in Bollettino di Studi Sardi, vol. 2, Cagliari, 2009, pp. 49-88.

Venturi, F (1964), "Il conte Bogino, il dott. Cossu e i monti frumentari. Episodio di storia sardopiemontese del secolo XVIII", Rivista storica italiana, LXXVI, 1964, 2, pp. 470-506

Virdis, A. (1991) "Alcune figure del clero sardo nei moti rivoluzionari della fine del Settecento attraverso gli atti processuali dell'Archivio Arcivescovile di Sassari” in Sotgiu, G. / Accardo, A. / Carta, L., Intellettuali e società in Sardegna tra Restaurazione e Unità d'Italia, II vol., Oristano, S'Alvure, pp. 245-329.

Virdis, M. (2006), introduzione a Araolla, G.[1597], Rimas diversas spirituales, introduzione, edizione critica e commento di Virdis, M., Cagliari, Centro Studi Filologici sardi / CUEC, pp. ICLXXV.

. (2014) "Matteo Madao e la questione della lingua sarda" in QB Quaderni Bolotanesi, 40, 2014, pp. $75-92$. 